\title{
Synthesis of European pharmacopoeial impurities A, B, $C$, and D of cabergoline $\uparrow$
}

Received 4th July 2013

Accepted 24th September 2013

DOI: $10.1039 / \mathrm{c} 3 \mathrm{ra} 43417 f$

www.rsc.org/advances

\author{
Jernej Wagger, ${ }^{\text {*a }}$ Aljaž Požes ${ }^{\mathrm{b}}$ and Franc Požgan ${ }^{\mathrm{bc}}$
}

For the use of analytics, European pharmacopoeial impurities A, B, C, and D of cabergoline were synthesized. Ergocryptine was chosen as a starting material and synthesis was accomplished via two approaches, different in length and stereochemical outcome. A longer, indirect approach was realized through otherwise problematic oxidations of the 9,10-dihidrolysergol derivative, to the corresponding aldehyde and carboxylic acid. This was achieved by the use of activated DMSO and a Pinnick oxidation sequence. All four synthesized impurities are used as analytical standards in cabergoline manufacturing processes.

\section{Introduction}

Cabergoline (1) is a natural product-based drug, representing a complex, branched amide of 6- $\mathrm{N}$-allylated 9,10-dihydrolysergic

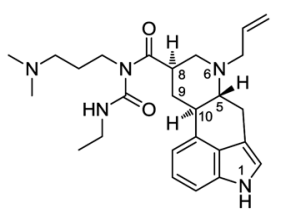<smiles></smiles>
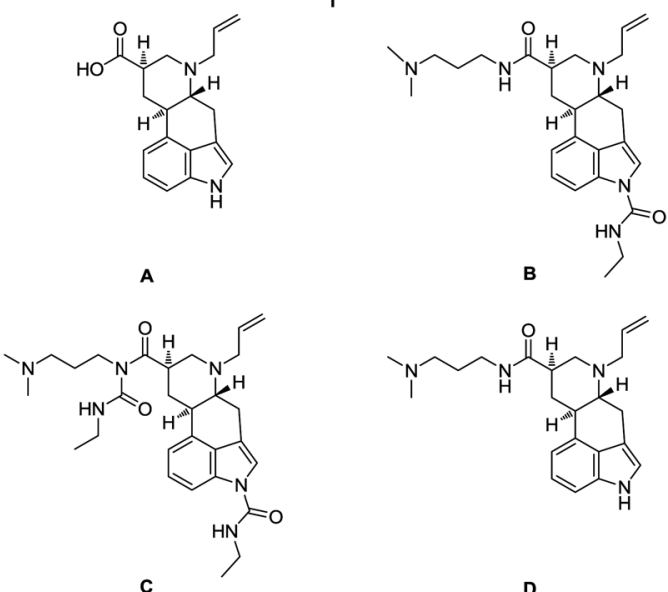

D
${ }^{a}$ Lek d.d., a Sandoz Company, Kolodvorska cesta 27, 1234 Mengeš, Slovenia. E-mail: jernej.wagger@sandoz.com; Tel: +386 17217206

${ }^{b}$ Faculty of Chemistry and Chemical Technology, University of Ljubljana, Aškerčeva 5, 1000 Ljubljana, Slovenia

${ }^{c}$ EN $\rightarrow$ FIST Centre of Excellence, Dunajska c. 156, 1000 Ljubljana, Slovenia

$\dagger$ Electronic supplementary information (ESI) available: Copies of ${ }^{1} \mathrm{H}$ and ${ }^{13} \mathrm{C}$ NMR spectra of all synthesised compounds. See DOI: 10.1039/c3ra43417f acid (cabergolinic acid) A (Fig. 1). Primarily acting as a dopaminergic $\mathrm{D}_{2}$ receptor agonist, cabergoline is most widely used for treatment of hyperprolactinaemic disorders and both early and advanced Parkinson's disease. ${ }^{1}$ The structure of cabergoline also encodes for different biogenic amines than dopamine, therefore displaying modest pharmacodynamic properties towards adrenergic and serotonergic receptors. ${ }^{1}$

One of the fundamental roles of the pharmaceutical industry is to develop new drugs that are safe, effective and of high quality when they reach the patients. With respect to this, delivering an impurity profile of an active pharmaceutical ingredient (API) is a must for fulfilling the aforementioned criteria. ${ }^{2}$ For the purpose of qualifying and/or quantifying the impurity profile of cabergoline (1) as the API, we have synthesized four known impurities, qualified by European Pharmacopoeia (Ph. Eur.) ${ }^{3}$ as impurities A, B, C and $\mathbf{D}$ (Fig. 1).

The genesis of these impurities can be found in the syntheses and other manufacturing process activities of cabergoline (Scheme 1). ${ }^{4}$

Since cabergoline (1), with the maximum weekly dosage of $2.0 \mathrm{mg}$, falls into low dosing drugs, the threshold for identification and qualification of impurities in API is usually $0.10 \%$ and $0.15 \%$ of mass, respectively. ${ }^{2}$ These limits are low and efficient production processes should not exceed them. Therefore production syntheses are usually neither suitable nor cost

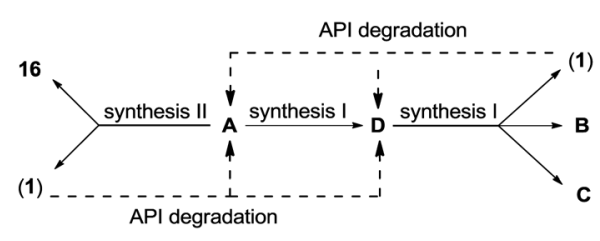

Scheme 1 Possible routes of cabergoline impurities formation. Some impurities (A, D) can represent syntheses intermediates or are formed via hydrolysis of amide bonds from cabergoline (1) or other impurities (B, C). 
effective means to produce impurities for the use of analytics. Often, new synthesis routes have to be designed to obtain desired impurities and this was also our task. Synthesis routes for Ph. Eur. impurities A, B, C and D of cabergoline (1) were designed and results are presented in this paper.

\section{Results and discussion}

9,10-Dihydrolysergic acid (2) was selected as one of the key synthetic intermediates. Although there are several total syntheses of lysergic acid available, through which 9,10-dihydrolysergic acid (2) could be prepared, $\alpha$-ergocryptine (3) was chosen as starting material. The reason is that $\alpha$-ergocryptine (3) is biosynthetically produced at our production site and way of preparing 9,10-dihydrolysergic acid (2) from $\alpha$-ergocryptine (3) is advantageous over total synthesis because it is shorter, easier and more economical. Also, there is a longstanding tradition of ergot alkaloids chemistry within Sandoz company, pioneered by Albert Hofmann.

Starting with $\alpha$-ergocryptine (3), the double bond between C9-C10 was diastereoselectively hydrogenated and the reduced ergopeptine 4 was cleaved by alkaline hydrolysis to give 9,10dihydrolysergic acid (2). ${ }^{5}$ To increase solubility of 9,10-dihydrolysergic acid (2) in organic solvents, the corresponding methyl ester 5 was prepared (Scheme 2). ${ }^{6}$

In the next steps allylic substituent on $6-N$ of 5 had to be introduced. To introduce the allylic substituent on $6-N$ of 5 , 9,10-dihydrolysergic acid (2) had to be $N$-demethylated first. 2,2,2Trichloroethyl chloroformate (TrocCl) was used, in a transformation mechanistically analogues to the von Braun reaction, ${ }^{7}$ to $N$-demethylate 9,10-dihydrolysergic acid methyl ester (5). Subsequent zinc mediated reductive cleavage of 6-N-Troc substituted compound 6 gave the desired 6 - $N$-demethylated intermediate 7. This $N$-demethylation sequence was already reported earlier and slight procedure modifications were used in our case. ${ }^{8}$ We have noticed that intermediate 7 decomposes slowly in acetic acid and is photolabile and therefore, the reaction
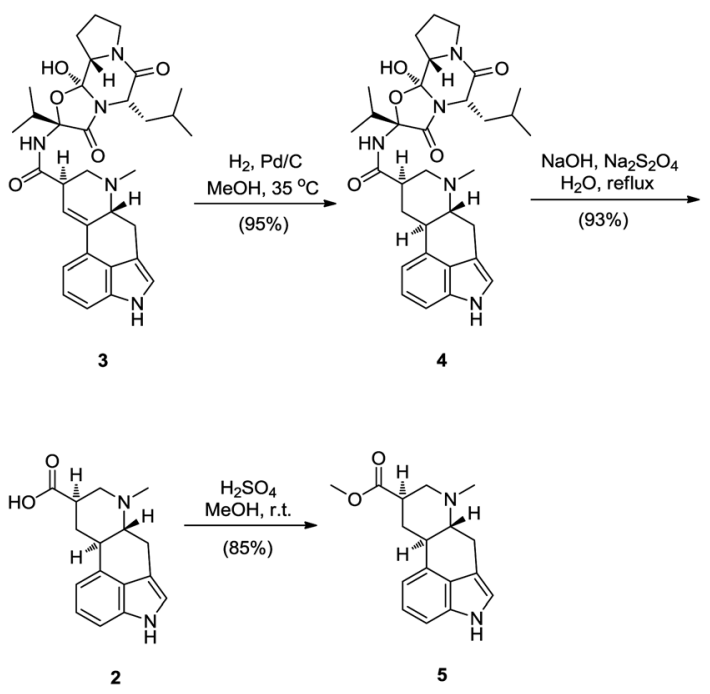

Scheme 2 Synthesis of 9,10-dihydrolysergic acid methyl ester $\mathbf{5}$ yield depends on the swiftness of work-up and isolation. 6- $\mathrm{N}$ Allylation of 7 was then realized by reaction with allylbromide and potassium carbonate in dimethylformamide, giving allylated intermediate 8 in a high yield (Scheme 3 ). ${ }^{9}$

At this point, intermediate 8 served us for the synthesis of impurities $\mathbf{A}$ and D. Alkaline hydrolysis of methyl ester $\mathbf{8}$ gave cabergolinic acid, termed as impurity A by Ph. Eur., in $82 \%$ yield. The impurity $\mathbf{D}$ was prepared directly from methyl ester $\mathbf{8}$ as well. By reacting 8 with 3-(dimethylamino)-1-propylamine in acetic acid, direct amidation of ester gave impurity $\mathbf{D}$ in $74 \%$ yield (Scheme 4$){ }^{4}$

Compared to $\mathbf{A}$ and $\mathbf{D}$, both impurities $\mathbf{B}$ and $\mathbf{C}$ have an ethylcarbamoyl functionality on the indole nitrogen. Since such functionalization of indole nitrogen requires the use of base, epimerization at the $\mathrm{C} 8$ position of ester 8 was possible. Although the stereocenter at C8 in 9,10-dihydrolysergic acid (2) is less prone to epimerization under basic conditions than in lysergic acid, due to the absence of C9-C10 double bond, epimerization does occur. ${ }^{\mathbf{1 0 , 1 1}}$ For example, it is known from the literature, that exposing compound 5 to 4 equivalents of $\mathrm{NaH}$ in THF at $20{ }^{\circ} \mathrm{C}$, app. $30 \%$ of the opposite, thermodynamically less favourable, axial epimer is observed, and by exposing compound 8 to 4 equivalents of LDA in THF at $-20{ }^{\circ} \mathrm{C}$, almost
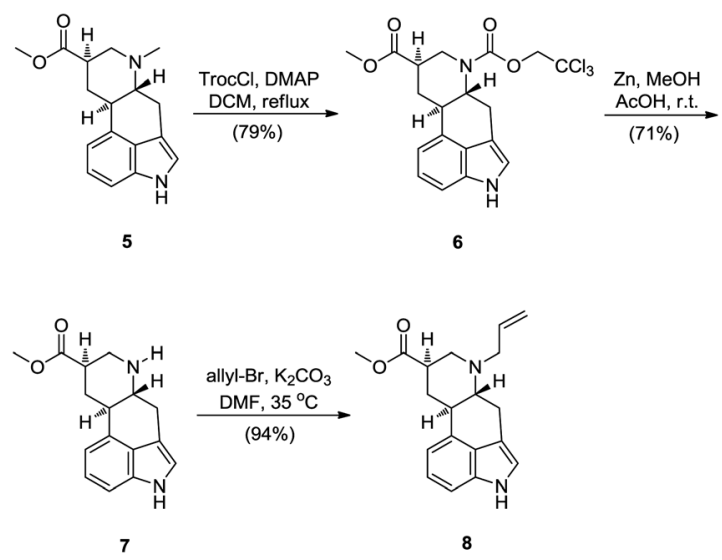

Scheme 3 Synthesis of 6-N-allylated 9,10-dihydrolysergic acid methyl ester 8
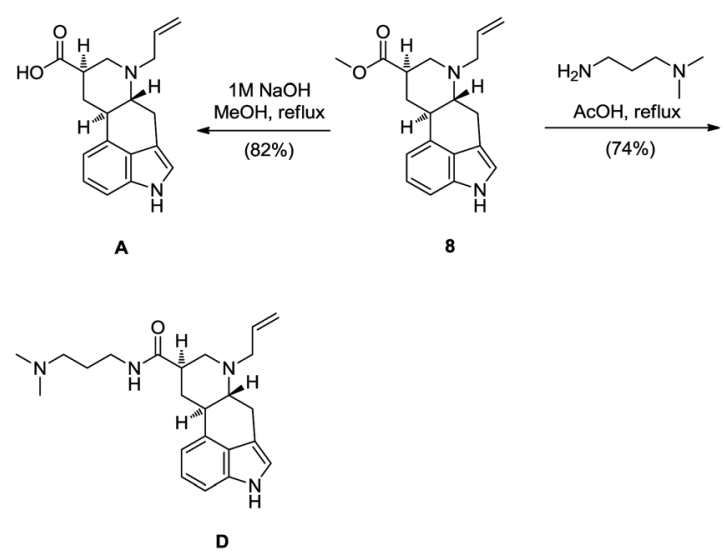

Scheme 4 Synthesis of Ph. Eur. impurities A and D from intermediate 8. 
$71 \%$ of axial epimer is generated. ${ }^{11}$ Therefore, for installing the ethylcarbamoyl fragment on the indole nitrogen $N-1$ we have envisioned two different approaches. In the first one, a direct functionalization of $N$-1 of $\mathbf{8}$, under basic conditions with EtNCO was planned. Being aware of the potential epimerisation problems under these conditions, a second indirect approach was envisioned. In this, indirect approach it was planned to reduce $\mathrm{C} 8$ carbonyl group of $\mathbf{8}$ to corresponding alcohol, do the functionalization of $N-1$ with EtNCO under basic conditions and then reoxidise the alcohol back to the corresponding carboxylic acid. Although longer, this reduction/oxidation approach, in comparison to the first direct one, would ensure us with the epimerisation free preparation of the desired carboxylic acid. Since both approaches for functionalization of $N-1$, direct and indirect one, have advantages and disadvantages, we have decided to test both of them. Subjecting methyl ester 8 to the 1.1 equivalents of $\mathrm{NaH}$ and EtNCO afforded the desired epimer 9a in $6: 1$ diastereomeric ratio and with $47 \%$ isolated yield (Scheme 5). ${ }^{12}$ With the methyl ester 9a in hand we were only one step short of the key intermediate 9,10-dihydrolysergic acid 10, which would enable us to prepare impurities $\mathbf{B}$ and $\mathbf{C}$. Luckily saponification of the methyl ester 9a proceeded selectively with respect to the $N-1$ ethylcarbamoyl fragment. This has provided us with the desired 9,10-dihydrolysergic acid derivative 10 in $77 \%$ yield. We found 9,10-dihydrolysergic acid 10 rather difficult to isolate, since it displays modest solubility in both organic solvents and water, but with acid $\mathbf{1 0}$ in hand, the door towards completion of the synthesis plan was open (Scheme 5).

For the indirect approach, methyl ester $\mathbf{8}$ had to be reduced first. Reacting ester 8 with the $\mathrm{LiAlH}_{4}$ in THF proceeded smoothly and in high yield to give 9,10-dihydrolysergol derivative $11 .{ }^{13}$ Hydroxy group in 9,10-dihydrolysergol derivative 11 was protected as TBDMS ether $\mathbf{1 2}$ and a carbamoyl moiety was introduced by reacting 12 with sodium hydride and ethylisocyanate to give TBDMS-protected 9,10-dihydrolysergol derivative 13 (Scheme 6).
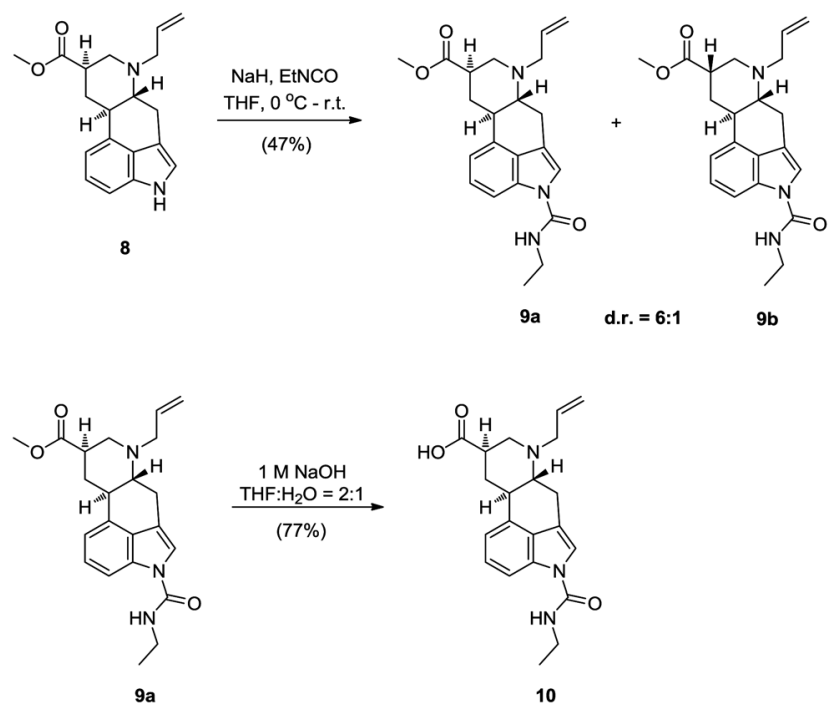

Scheme 5 Direct synthesis of the key 9,10-dihydrolysergic acid 10-functionalization of the 9,10-dihydrolysergic acid methyl ester $\mathbf{8}$.

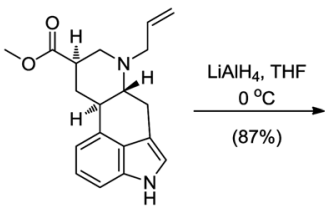

8

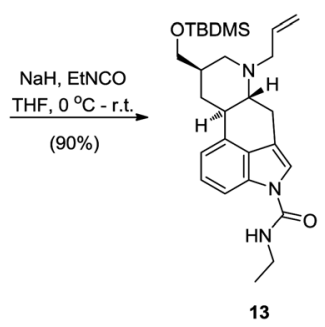

Scheme 6 Synthesis of $\mathbf{1 3}$-introduction of an ethylcarbamoyl fragment onto the indole nitrogen of lysergol derivative 12.

For the next steps, TBDMS-protected alcohol group in $\mathbf{1 3}$ had to be removed and free alcohol reoxidized back to carboxylic acid. Deprotection of silyl ether in 13 with TBAF gave 9,10dihydrolysergol derivative $\mathbf{1 4}$ in very good yield, although some temperature dependent chemoselectivity was observed. When silyl deprotection was performed at $0{ }^{\circ} \mathrm{C}$, slow but clean conversion of $\mathbf{1 3}$ to $\mathbf{1 4}$ was achieved. On the other hand, when the reaction was done at room temperature, removal of the ethylcarbamoyl group was observed in addition to silyl deprotection. 9,10-Dihydrolysergol derivative $\mathbf{1 4}$ was then used for subsequent oxidation reaction. Ideally a one-step oxidation procedure from primary alcohol $\mathbf{1 4}$ to the corresponding carboxylic acid would be a method of choice. But surprisingly, while searching the literature, we learned that examples covering oxidations of 9,10-dihydrolysergols and their derivatives to the corresponding 9,10-dihydrolysergals and/or 9,10dihydrolysergic acids are very scarce. In the most recent literature, Křen et al. reported on their efforts to oxidize $N$-1-protected lysergol derivatives to aldehydes and acids..$^{14}$ Beside that, patent literature covers two examples of 9,10-dihydrolysergol oxidations. In the first one BAIB/TEMPO was used for direct oxidation of 9,10-dihydrolysergol derivative to the corresponding 9,10dihydrolysergic acid..$^{15}$ Unfortunately, BAIB/TEMPO mediated oxidation did not work in our case, giving only traces of the desired acid, and failure to oxidize the alcohol $\mathbf{1 4}$ is in agreement with the results reported in the literature..$^{14}$ In the second literature example, slightly modified Parikh-Doering oxidation $\left(\mathrm{SO}_{3} \cdot \mathrm{TEA}, \mathrm{DMSO}, \mathrm{TEA}\right)$ was successfully used to oxidize 9,10dihydrolysergol to the 9,10 -dihydrolysergal, ${ }^{16}$ but as reported by Křen, they were unsuccessful in repeating this type of oxidation under standard Parikh-Doering conditions $\left(\mathrm{SO}_{3} \cdot \mathrm{Py}\right.$, DMSO, TEA). ${ }^{14}$ Since 9,10-dihidrolysergol derivatives are poorly soluble in various solvents, we anticipated that DMSO mediated oxidations could be a good choice amongst the plethora of alcohol oxidation methods. DMSO should address the solubility issue of 9,10-dihydrolysergol derivative and concurrently serve as an oxidant. Since contrary reports about effectiveness of the Parikh-Doering oxidation of 9,10-dihidrolysergols were 
available in the literature, we have decided to try other analogues reaction. Luckily one can chose among different DMSO mediated oxidations. Indeed, when alcohol 14 was subjected to Pfitzner-Moffatt oxidation conditions, a clean and high yielding conversion to the corresponding 9,10-dihydrolysergal 15 was observed. ${ }^{17}$ Here again temperature dependence on reaction selectivity was observed. Below $5{ }^{\circ} \mathrm{C}$ the reaction was clean, giving only aldehyde as a product, while at room temperature or higher several side products could be observed by TLC. Since we were successful with Pfitzner-Moffatt oxidation of 14, the question was raised, whether the outcome of Parikh-Doering reaction, on the same alcohol substrate would be analogues? Oxidation of the alcohol 14, under the Parikh-Doering conditions, proceeded smoothly, affording aldehyde 15 in slightly lower yield than via Pfitzner-Moffatt reaction (Scheme 7). ${ }^{18}$

An added value of this oxidation transformations also lies in the 9,10-dihydrolysergal itself. Knowing that aldehydes of this type are difficult to prepare and that pharmacological activity of synthetically modified ergot alkaloids varies mainly because of the nature and configuration of substituents on $\mathrm{C} 8$, such aldehydes also represent valuable medicinal chemistry intermediates. $^{4 a, 16 a, 19}$

Oxidations of 9,10-dihydrolysergals to the corresponding 9,10dihydrolysergic acids are hardly reported in the literature. This is mainly due to the fact that 9,10-dihydrolysergic acid derivatives are easily accessible from naturally occurring lysergic acid. The literature reports on oxidation of 9,10-dihydrolysergal with Tollens's reagent or MCPBA, but both methods have their limitations. ${ }^{14}$ The first one unfortunately gives poor yields while the second one suffers from chemoselectivity. Peroxy acid oxidizes not only the aldehyde, but also the nitrogen on position 6 to the corresponding $\mathrm{N}$-oxide. Although reduction of $\mathrm{N}$-oxide to the desired 9,10-dihydrolysergic acid was demonstrated, ${ }^{\mathbf{1 4}}$ this method suffers from functional group compatibility, since allylated lysergal derivatives are not tolerated by peroxy acids nor
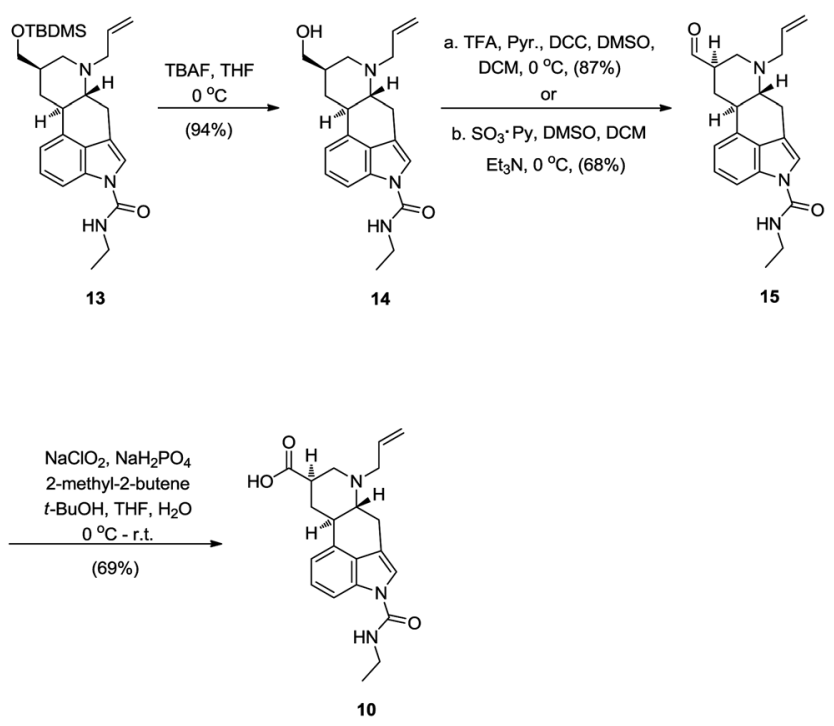

Scheme 7 Indirect, oxidative route to the 9,10-dihydrolysergic acid $\mathbf{1 0}$. catalytic hydrogenation. On the other hand there are some examples of lysergal oxidations to lysergic acid derivatives. In the final steps of total synthesis of lysergic acid by Fujii and Ohno, Pinnick oxidation was used to oxidize 1- $\mathrm{N}-6-\mathrm{N}$-ditosylated isolysergal into the corresponding lysergic acid. ${ }^{20}$ Indeed, upon subjecting aldehyde 15 to Pinnick oxidation, the desired 9,10dihydrolysergic acid 10 was obtained, in 69\% yield (Scheme 7). ${ }^{21}$

9,10-Dihydrolysergic acid 10 was then used to synthesize both impurities $\mathbf{B}$ and $\mathbf{C}$. The coupling of 10 with 3-(dimethylamino)-1-propylamine by the use of CDI furnished the impurity $\mathrm{B}$ in $54 \%$ yield. For the synthesis of impurity $\mathrm{C}$ a more complex $\mathrm{N}$-acylurea appendage on $\mathrm{C} 8$ had to be installed. In the original synthesis of cabergoline, this side chain was installed with the use of EDC $\cdot \mathrm{HCl}$, by exploiting the usually undesired ability of carbodiimide coupling reagents to form $\mathrm{N}$-acylureas as intramolecular rearrangement side products. ${ }^{22}$ Reacting cabergolinic acid $\mathbf{A}$ with $\mathrm{EDC} \cdot \mathrm{HCl}$, in the absence of an external nucleophile, Mantegani and co-workers synthesized cabergoline (1). ${ }^{4}$ Although the use of $\mathrm{EDC} \cdot \mathrm{HCl}$ gives the desired $\mathrm{N}$-acylurea appendage in one step, a disadvantage of using this asymmetric carbodiimide is concomitant formation of regioisomeric $\mathrm{N}$-acylurea and tedious separation of both isomers.

To compensate for disadvantage of applying $\mathrm{EDC} \cdot \mathrm{HCl}$, different synthetic strategy was utilized in which excess ethylisocyanate was reacted with compound $\mathbf{D}$ to install $\mathrm{N}$-acylurea appendage. Unfortunately the use of ethylisocyanate is also the root cause for formation of impurities $\mathbf{B}$ and $\mathbf{C}$ as side products in cabergoline (1) synthesis (Scheme 1). ${ }^{4}$ Other methods for $\mathrm{N}$-acylurea installation in cabergoline (1) synthesis were also developed, although none of them introduces $N$-acylurea appendage in one step from the corresponding 9,10-dihydrolysergic acid. ${ }^{23}$ Therefore, compromising lower yield, we have used $\mathrm{EDC} \cdot \mathrm{HCl}$ in $\mathrm{DCM}^{24}$ for the synthesis of impurity $\mathbf{C}$, thus preparing the desired impurity and its regioisomer 16 in ratio 5:1 respectively. ${ }^{25}$ After separation of regioisomers, the impurity $\mathbf{C}$ was obtained in $58 \%$ yield (Scheme 8 ).

For the synthesis of $\mathbf{C}$, another synthetic strategy would be viable, by following linear route $\mathbf{1 0} \rightarrow \mathbf{B} \rightarrow \mathbf{C}$. By reacting $\mathbf{B}$ with an excess of EtNCO, impurity $\mathbf{C}$ could be prepared. In the original synthesis of cabergoline (1) up to 40 equivalents of EtNCO were reacted with compound $\mathbf{D}$ to get the desired API. The amount of EtNCO was later reduced in consequent synthesis optimizations, by the use of $\mathrm{CuI}$ and $\mathrm{Ph}_{3} \mathrm{P}$ catalysis, to as low as 3 equivalents. Under these, optimised conditions cabergoline (1) was prepared along with the impurities $\mathbf{B}$ and $\mathbf{C}$ in the ratio of $82: 6: 12$ respectively. ${ }^{26}$ As mentioned earlier, other syntheses of cabergoline (1) were developed to avoid the use of toxic EtNCO and they are all based on the functionalization of the carboxamide side chain of $\mathbf{D .}^{23}$ In our case the impurity $\mathbf{B}$ was prepared from precursor 10 in a modest 54\% yield. Therefore, if the reaction of $\mathbf{B}$ with EtNCO, or any other suitable reagent, would work quantitatively, the impurity $\mathbf{C}$, starting linearly from 10, would be prepared in not greater than $54 \%$ yield. Following this, we have never tried to prepare impurity $\mathbf{C}$ from $\mathbf{1 0}$ via impurity $\mathbf{B}$. Instead, the impurity $\mathbf{C}$ was prepared in one step, directly from carboxylic acid 10 with 58\% yield (Scheme 8). 

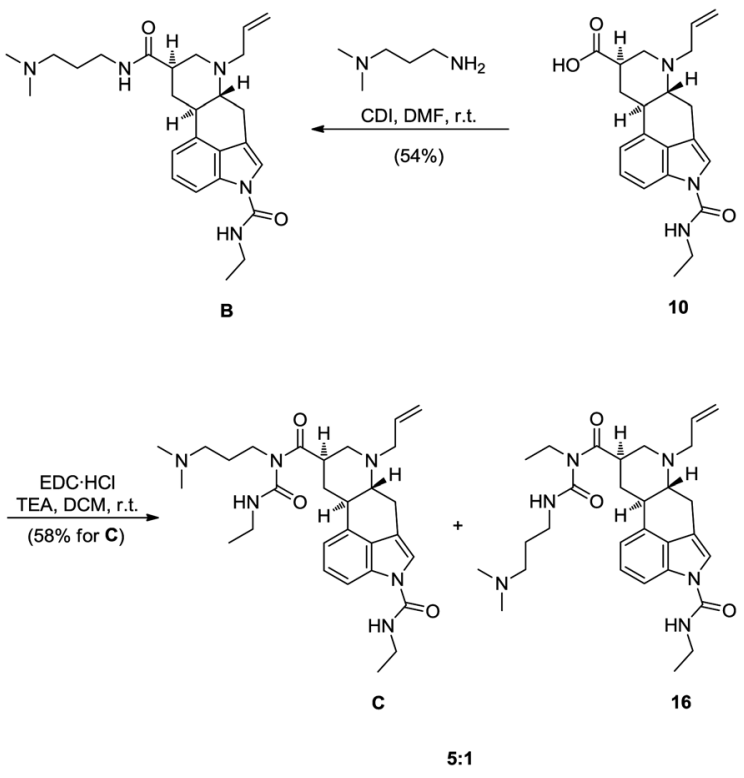

Scheme 8 Synthesis of Ph. Eur. impurities B and C.

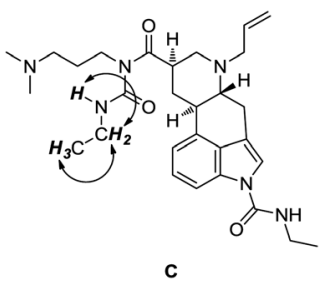

Fig. 2 Detected COSY interactions in ethylamido side chain $\left(\mathrm{CH}_{3}, 1.18 \mathrm{ppm} ; \mathrm{CH}_{2}\right.$, 3.30 ppm; NH, 9.45 ppm) in impurity $\mathbf{C}$ are indicated by arrows.

The structure of proper $N$-acylurea appendage in the impurity C was determined using 2D COSY NMR technique, where expected interactions between ethylamido protons were observed (Fig. 2). The proton NMR of the impurity $\mathbf{C}$ is also in accordance with the previous report of Mantegani and co-workers. ${ }^{4}$

\section{Conclusions}

A synthesis of four European pharmacopoeial impurities A, B, C and $\mathbf{D}$ of cabergoline (1) was demonstrated and the synthesized impurities are used as HPLC analytical standards. To the best of our knowledge, this is the first report on selective synthesis of these impurities. From $\alpha$-ergocryptine (3), 9,10-dihydrolysergic acid methyl ester (5) was prepared, which served as the key starting material. Using 5, N-1 ethylcarbamoyl 9,10-dihydrolysergic acid $\mathbf{1 0}$ was prepared via two different routes. First route, a direct one, proceeds through direct $\mathrm{N}-1$ ethylcarbamoylation of 10, causing partial epimerisation at C8 position. In spite of epimerisation, this route is shorter and therefore advantageous over the indirect one. Second route, an indirect one, follows reduction-oxidation of 9,10-dihydrolysergic acid derivative $\mathbf{8}$, for epimerisation free installation of $N$-1 ethylcarbamoyl moiety. Notwithstanding the fact that oxidations of 9,10-dihydrolysergol derivatives to the corresponding 9,10-dihydrolysergals and further on to 9,10dihydrolysergic acids are very rare, we have shown that by combination of activated DMSO and Pinnick reactions this oxidation sequence can be achieved successfully. Furthermore, such oxidation method of preparing otherwise scarcely presented 9,10-dihydrolysergals, can be useful in medicinal chemistry, for preparing novel pharmacologically active C8substituted derivatives of ergoline.

\section{Experimental}

\section{General experimental details}

Where stated, anhydrous reactions were carried out in vacuum oven dried glassware, under stream of nitrogen, passed through Drierite $^{\mathrm{TM}}$ drying column. Anhydrous solvents were purchased form Sigma-Aldrich and all reagents were commercial grade, used without any purification. Exception was $\alpha$-ergocryptine which was acquired through in-house biofermentation process and was purified by crystallization from toluene. Reactions were monitored by thin layer chromatography (TLC) performed on pre-coated aluminum-baked silica gel (60 $\mathrm{F}_{254}$, Merck) or aluminum oxide $\left(60 \mathrm{~F}_{254}\right.$, Merck) plates. Developed TLC plates were visualized under UV light and/or by aqueous cerium ammonium molybdate and aqueous potassium permanganate. Flash chromatography was performed on ZEOPrep 60 Eco 40-63 $\mu \mathrm{m}$ silica gel and aluminum oxide activated, basic, with indicated mobile phase. Lower-temperature reactions were performed on ice/water bath or on Mettler-Toledo EasyMax ${ }^{\mathrm{TM}}$ synthesis workstation. Infrared spectra were recorded on a Bruker Alpha (Platinum ATR) FT-IR spectrometer and are reported in reciprocal centimeters $\left(\mathrm{cm}^{-1}\right)$. Nuclear magnetic resonance (NMR) spectra were recorded on a Bruker Avance III 500 and Bruker Avance DPX 300 spectrometers. Chemical shifts for ${ }^{1} \mathrm{H}$ NMR spectra are reported in parts per million from tetramethylsilane, with the solvent resonance as the internal standard $\left(\mathrm{CDCl}_{3}, \delta 7.26 \mathrm{ppm}, \mathrm{DMSO}-d_{6}, \delta 2.50 \mathrm{ppm}\right)$. Data are reported as follows: chemical shift, multiplicity, ( $\mathrm{s}=$ singlet, $\mathrm{d}$ $=$ doublet, $\mathrm{t}=$ triplet, $\mathrm{q}=$ quartet, $\mathrm{qn}=$ quintet, $\mathrm{m}=$ multiplet, $\mathrm{br}=$ broad), coupling constant in $\mathrm{Hz}$ and integration. Chemical shifts for ${ }^{13} \mathrm{C}$ NMR spectra are reported in parts per million from tetramethylsilane, using solvent central peak as the internal standard $\left(\mathrm{CDCl}_{3}, \delta 77.16 \mathrm{ppm}, \mathrm{DMSO}-d_{6}, \delta 39.52 \mathrm{ppm}\right)$. Where stated 2D NMR spectra were recorded. For $\mathrm{pH}$ measurements of water solutions, Seven Easy Mettler-Toledo pH meter was used. Optical rotations were determined at 589 $\mathrm{nm}$ at ambient temperature on Perkin-Elmer 2400 241MC polarimeter. Data reported as follows: $[\alpha]_{\mathrm{D}}$, concentration $(c$ in $\mathrm{g}$ per $100 \mathrm{~mL}$ ) and solvent. Elemental microanalyses were performed on Perkin-Elmer Analyser 2400. Mass spectra were obtained using an AutoSpecQ, Q-TOF Premier and Agilent 6224 Accurate TOF LC/MS spectrometers. Melting points were determined on a Kofler micro hot stage and are uncorrected.

9,10-Dihydro- $\alpha$-ergocryptine (4). In a $1000 \mathrm{~mL}$ round-bottom flask $\alpha$-ergocryptine (3) was weighed (30 g, $52 \mathrm{mmol}$ ) and dissolved in $\mathrm{MeOH}(500 \mathrm{~mL})$ at $35{ }^{\circ} \mathrm{C}$. Then, while stirring, $\mathrm{Pd}$ (3.9 $\mathrm{g}, 10 \%$ on carbon) suspended in $\mathrm{MeOH}(143 \mathrm{~mL})$ was added and reaction mixture was purged with $\mathrm{N}_{2}$. After that reaction 
mixture was evacuated, purged with $\mathrm{H}_{2}$ and put under $\mathrm{H}_{2}$ atmosphere (balloon). Reaction mixture was stirred at $35^{\circ} \mathrm{C}$ for $3 \mathrm{~h}$ and after that it was purged with $\mathrm{N}_{2}$ and heated to $55^{\circ} \mathrm{C}$. At $55{ }^{\circ} \mathrm{C}$ the Pd catalyst was filtered off and filtrate was ran through aluminum oxide (30 g, basic) column and column was washed with $\mathrm{MeOH}(2 \times 200 \mathrm{~mL})$ heated to $55{ }^{\circ} \mathrm{C}$. Combined $\mathrm{MeOH}$ fractions were evaporated at reduced pressure to dryness and resuspended in $\mathrm{MeOH}(450 \mathrm{~mL})$. Suspension was heated to $55{ }^{\circ} \mathrm{C}$ and while stirring, $\mathrm{H}_{2} \mathrm{O}(900 \mathrm{~mL})$ was added. Stirred suspension was cooled to ambient temperature and further to $0{ }^{\circ} \mathrm{C}$, at which it was stirred for $0.5 \mathrm{~h}$. Precipitate, was filtered off and dried in vacuum to give the titled compound as an off white solid (28.5 g, 95\%). Optionally, product could be further crystallized from MeOH. $R_{\mathrm{f}}: 0.33$ (EtOAc-MeOH $=90 \%: 10 \%$ ); $\mathrm{mp}$ $=224-226{ }^{\circ} \mathrm{C}$, ref. $5 a=235{ }^{\circ} \mathrm{C}$; $[\alpha]_{\mathrm{D}}-30.1$ (c 0.53; pyr.), ref. $5 a$ $[\alpha]_{\mathrm{D}}-41$ (Pyr.); IR: $\nu_{\max } 3627,3369,3354,3198,3064,2955$, 2847, 2792, 1720, 1657, 1626, 1546, 1432, 1218, 1191, 1003, 755 $\mathrm{cm}^{-1} ;{ }^{1} \mathrm{H}$ NMR (DMSO- $\left.d_{6}, 500 \mathrm{MHz}\right) \delta 0.86(\mathrm{~d}, J=6.7 \mathrm{~Hz}, 3 \mathrm{H})$; 0.90 (d, $J=6.8 \mathrm{~Hz}, 3 \mathrm{H}) ; 0.94$ (d, $J=6.5 \mathrm{~Hz}, 3 \mathrm{H}) ; 1.05$ (d, $J=6.7$ $\mathrm{Hz}, 3 \mathrm{H}) ; 1.40$ (q, $J=12.4 \mathrm{~Hz}, 1 \mathrm{H}) ; 1.64-1.82$ (m, 3H); 1.85-2.05 $(\mathrm{m}, 5 \mathrm{H}) ; 2.12\left(\mathrm{dt}, J_{1}=13.5 \mathrm{~Hz}, J_{2}=6.7 \mathrm{~Hz} ; 1 \mathrm{H}\right) ; 2.22(\mathrm{t}, J=12.2$ $\mathrm{Hz}, 1 \mathrm{H}) ; 2.36(\mathrm{~s}, 3 \mathrm{H}) ; 2.48-2.54(\mathrm{~m}, 1 \mathrm{H}) ; 2.63(\mathrm{bd}, J=12.2 \mathrm{~Hz}$, $1 \mathrm{H}) ; 2.78-2.84(\mathrm{~m}, 1 \mathrm{H}) ; 2.94-3.02(\mathrm{~m}, 2 \mathrm{H}), 3.30\left(\mathrm{dd}, J_{1}=14.7 \mathrm{~Hz}\right.$, $\left.J_{2}=4.1 \mathrm{~Hz}, 1 \mathrm{H}\right) ; 3.35-3.41(\mathrm{~m}, 2 \mathrm{H}) ; 3.72\left(\mathrm{dd}, J_{1}=8.4 \mathrm{~Hz}, J_{2}=5.9\right.$ $\mathrm{Hz}, 1 \mathrm{H}) ; 4.32\left(\mathrm{dd}, J_{1}=7.6 \mathrm{~Hz}, J_{2}=5.8 \mathrm{~Hz}, 1 \mathrm{H}\right) ; 6.74(\mathrm{~d}, J=7.02$ $\mathrm{Hz}, 1 \mathrm{H}) ; 6.98$ (bs, 1H); 7.02 (t, $J=7.9 \mathrm{~Hz}, 1 \mathrm{H}) ; 7.13$ (d, $J=8.1 \mathrm{~Hz}$; $1 \mathrm{H}) ; 7.57$ (d, $J=1.58 \mathrm{~Hz}, 1 \mathrm{H}) ; 9.05$ (s, $1 \mathrm{H}) ; 10.66$ (d, $J=1.55 \mathrm{~Hz}$, 1H). ${ }^{13} \mathrm{C}$ NMR (DMSO- $\left.d_{6}, 125 \mathrm{MHz}\right) \delta 15.7,16.6,21.5,22.2,22.7$, 24.3, 26.0, 26.6, 30.5, 33.0, 39.4, 41.8, 42.6, 43.0, 45.7, 52.4, 58.8, 63.5, 66.5, 90.15, 103.3, 108.9, 110.0, 111.7, 118.6, 122.1, 125.9, 132.16, 133.20, 164.9, 165.2, 177.1; HRMS (ESI): $\mathrm{m} / \mathrm{z}$ calcd for $\mathrm{C}_{32} \mathrm{H}_{44} \mathrm{~N}_{5} \mathrm{O}_{5}:[\mathrm{M}+\mathrm{H}]^{+}, 578.3337$; found: 578.3337 .

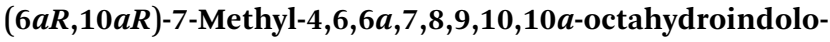
[4,3-fg]quinoline-9-carboxylic acid (2). To $\mathrm{NaOH}(8.28 \mathrm{~g}, 207$ $\mathrm{mmol})$ in $250 \mathrm{~mL}$ flask, $\mathrm{H}_{2} \mathrm{O}(96 \mathrm{~mL})$ and $\mathrm{Na}_{2} \mathrm{~S}_{2} \mathrm{O}_{4}(1.2 \mathrm{~g}, 6.89$ $\mathrm{mmol}$ ) were added and solution was formed at room temperature. While stirring, 9,10-dihydro- $\alpha$-ergocryptine (4) (20 g, 34.6 $\mathrm{mmol}$ ) was suspended in the solution and reaction mixture was refluxed for $4 \mathrm{~h}$. After that, dark brown solution was cooled to room temperature and acidified with $\mathrm{AcOH}$ to $\mathrm{pH}$ 6.2. Suspension formed was cooled bellow $15{ }^{\circ} \mathrm{C}$ and stirred for $2 \mathrm{~h}$. Suspension was filtered off, thoroughly washed with $\mathrm{H}_{2} \mathrm{O}$ and dried to give 9,10-dihydrolysergic acid (2) as a light grayish solid $(8.7 \mathrm{~g}, 93 \%) . R_{\mathrm{f}}: 0.58$ (THF-AcOH- $\left.\mathrm{H}_{2} \mathrm{O}=75 \%: 13 \%: 12 \%\right) ; \mathrm{mp}$ $=$ decomposition at $250{ }^{\circ} \mathrm{C}$, ref. $5 a$ compound darkens at $250{ }^{\circ} \mathrm{C}$ and decomposes at $300{ }^{\circ} \mathrm{C} ;[\alpha]_{\mathrm{D}}-120\left(c 0.1\right.$; pyr.), ref. $5 a[\alpha]_{\mathrm{D}}$ -122 (c 0.2; Pyr.); IR: $\nu_{\max } 3370,3240,3194,3117,3048,2948$, 2852, 2348, 1577, 1445, 1390, 1345, 1090, 1029, 1007, $764 \mathrm{~cm}^{-1}$; ${ }^{1} \mathrm{H}$ NMR (DMSO- $\left.d_{6}, 500 \mathrm{MHz}\right) \delta 1.34(\mathrm{q}, J=12.9 \mathrm{~Hz}, 1 \mathrm{H}) ; 2.00$ $\left(\mathrm{dt}, J_{1}=10.9 \mathrm{~Hz}, J_{2}=4.1 \mathrm{~Hz}, 1 \mathrm{H}\right) ; 2.17(\mathrm{t}, J=11.5 \mathrm{~Hz}, 1 \mathrm{H}) ; 2.37$ (s, 3H); 2.48-2.55 (m, 1H); 2.70-2.85 (m, 3H); 3.12 (dd, $J_{1}=11.0$ $\left.\mathrm{Hz}, J_{2}=2.1 \mathrm{~Hz}, 1 \mathrm{H}\right) ; 3.29\left(\mathrm{dd}, J_{1}=14.6 \mathrm{~Hz}, J_{2}=4.1 \mathrm{~Hz}, 1 \mathrm{H}\right) ; 6.79$ $(\mathrm{d}, J=7.1 \mathrm{~Hz}, 1 \mathrm{H}) ; 6.98$ (bs, 1H); 7.01 (t, $J=7.6 \mathrm{~Hz}, 1 \mathrm{H}) ; 7.13$ (d, $J=8.1 \mathrm{~Hz}, 1 \mathrm{H}) ; 10.65$ (s, $1 \mathrm{H}) .{ }^{13} \mathrm{C}$ NMR (DMSO- $\left.d_{6}, 125 \mathrm{MHz}\right) \delta$ 26.5, 30.5, 40.9, 42.6, 58.6, 66.6, 108.8, 110.0, 112.0, 118.6, 122.1, 125.9, 132.3, 133.2, 175.1; HRMS (ESI): $\mathrm{m} / \mathrm{z}$ calcd for $\mathrm{C}_{16} \mathrm{H}_{19} \mathrm{~N}_{2} \mathrm{O}_{2}:[\mathrm{M}+\mathrm{H}]^{+}$, 271.1441; found: 271.1443 .
Methyl (6aR,10aR)-7-methyl-4,6,6a,7,8,9,10,10a-octahydroindolo $[4,3-f g]$ quinoline-9-carboxylate (5). To 9,10-dihydrolysergic acid (2) (7.00 g, $25.9 \mathrm{mmol}), \mathrm{MeOH}(125 \mathrm{~mL})$ was added and suspension was cooled to $\sim 3{ }^{\circ} \mathrm{C}$. Then slowly, concentrated $\mathrm{H}_{2} \mathrm{SO}_{4}(6.7 \mathrm{~mL})$ was added to the suspension. After addition of $\mathrm{H}_{2} \mathrm{SO}_{4}$, reaction mixture was allowed to warm up to room temperature and it was stirred for additional $15 \mathrm{~h}$. After that $\mathrm{MeOH}(58 \mathrm{~mL})$ was partly evaporated, $\mathrm{H}_{2} \mathrm{O}(226 \mathrm{~mL})$ was added and the solution was basified with $25 \%$ aqueous solution of $\mathrm{NH}_{4} \mathrm{OH}$ to $\mathrm{pH}$ 8.80. Suspension formed, was stirred at room temperature for $2 \mathrm{~h}$, after which it was filtered, washed with $\mathrm{H}_{2} \mathrm{O}$ and dried to give titled compound as an off white solid $(6.25 \mathrm{~g}$, $85 \%$ ). $R_{\mathrm{f}}: 0.45$ (EtOAc-MeOH $\left.=9: 1\right) ; \mathrm{mp}=189-190{ }^{\circ} \mathrm{C} ;[\alpha]_{\mathrm{D}}$ -66.1 ( c 0.98; DCM); IR: $\nu_{\max } 3142,3094,3002,2947,2864,2834$, 2804, 1729, 1608, 1443, 1238, 1096, 1025, $745 \mathrm{~cm}^{-1}$; ${ }^{1} \mathrm{H}$ NMR $\left(\mathrm{CDCl}_{3}, 500 \mathrm{MHz}\right) \delta 1.56-1.66(\mathrm{~m}, 1 \mathrm{H}) ; 2.21\left(\mathrm{ddd}, J_{1}=11.1 \mathrm{~Hz}, J_{2}\right.$ $\left.=9.4 \mathrm{~Hz}, J_{3}=4.3 \mathrm{~Hz}, 1 \mathrm{H}\right) ; 2.34-2,42(\mathrm{~m}, 1 \mathrm{H}) ; 2.52(\mathrm{~s}, 3 \mathrm{H}) ; 2.66-$ $2.75(\mathrm{~m}, 1 \mathrm{H}) ; 2.94-3.04(\mathrm{~m}, 3 \mathrm{H}) ; 3.26-3.32(\mathrm{~m}, 1 \mathrm{H}) ; 3.42\left(\mathrm{dd}, J_{1}=\right.$ $\left.14.7 \mathrm{~Hz}, J_{2}=4.3 \mathrm{~Hz}, 1 \mathrm{H}\right) ; 3.75(\mathrm{~s}, 3 \mathrm{H}) ; 6.89(\mathrm{t}, J=1.6 \mathrm{~Hz}, 1 \mathrm{H})$; 6.95-6.98 (m, 1H); 7.16-7.21 (m, 2H); 8.07 (s, 1H). ${ }^{13} \mathrm{C}$ NMR $\left(\mathrm{CDCl}_{3}, 125 \mathrm{MHz}\right) \delta 27.0,30.7,40.3,41.6,43.2,51.9,58.8,66.8$, 108.8, 111.9, 113.4, 118.0, 123.3, 126.2, 132.8, 133.5, 174.5; HRMS (ESI): $m / z$ calcd for $\mathrm{C}_{17} \mathrm{H}_{21} \mathrm{~N}_{2} \mathrm{O}_{2}:[\mathrm{M}+\mathrm{H}]^{+}, 285.1598$; found: 285.1595 .

9-Methyl 7-(2,2,2-trichloroethyl) (6aR,10aR)-6,6a,8,-9,10-,10ahexahydroindolo[4,3-fg] quinoline-7,9(4H)-dicarboxylate (6). To an oven dried glassware, dried compound 5 (8.51 g, $29.9 \mathrm{mmol})$ was weighed and anhydrous DCM $(200 \mathrm{~mL})$ was added under $\mathrm{N}_{2}$ atmosphere. Mixture was stirred and TrocCl $(18.7 \mathrm{~mL}, 136 \mathrm{mmol})$ and DMAP (0.162 g, $1.33 \mathrm{mmol}$ ) were added. Reaction mixture was heated to reflux and stirred for $16 \mathrm{~h}$. After indicated time reaction mixture was cooled to room temperature, filtered and filtrate was concentrated under reduced pressure. Residue on evaporation was purified by column chromatography (EtOAc) to give titled compound as a white solid $(10.54 \mathrm{~g}, 79 \%) . R_{\mathrm{f}}: 0.89$ (EtOAc); $\mathrm{mp}=21{ }^{\circ} \mathrm{C}$, ref. $7208-210^{\circ} \mathrm{C} ;[\alpha]_{\mathrm{D}}-60.8$ (c 0.53; DCM); IR: $\nu_{\max } 3382,2955,2847,1717,1598,1436,1374,1266,1187$, 1146, 1030, 812, 756, $719 \mathrm{~cm}^{-1} ;{ }^{1} \mathrm{H}$ NMR (DMSO- $\left.d_{6}, 500 \mathrm{MHz}\right) \delta$ $1.67(\mathrm{~m}, 1 \mathrm{H}), 2.74-2.81(\mathrm{~m}, 1 \mathrm{H}) ; 3.00-3.08$ (m, 2H); 3.23-3.33 (m, $2 \mathrm{H}) ; 3.63-3.69(\mathrm{~m}, 1 \mathrm{H}) ; 3.64(\mathrm{~s}, 3 \mathrm{H}) ; 3.77\left(\mathrm{dd}, J_{1}=14.2, J_{2}=6.2\right.$ $\mathrm{Hz}, 1 \mathrm{H}) ; 4.05\left(\mathrm{dd}, J_{1}=14.2, J_{2}=2.6 \mathrm{~Hz}, 1 \mathrm{H}\right) ; 4.84(\mathrm{~d}, J=12.4 \mathrm{~Hz}$, $1 \mathrm{H}) ; 4.87$ (d, $J=12.4 \mathrm{~Hz}, 1 \mathrm{H}) ; 6.81$ (d, $J=7.1 \mathrm{~Hz}, 1 \mathrm{H}) ; 7.00$ (bs, $1 \mathrm{H}) ; 7.03(\mathrm{~m}, 1 \mathrm{H}) ; 7.17$ (d, $J=8.1 \mathrm{~Hz}, 1 \mathrm{H}) ; 10.73$ (d, $J=1.5 \mathrm{~Hz}$, 1H). ${ }^{13} \mathrm{C}$ NMR (DMSO- $\left.d_{6}, 125 \mathrm{MHz}\right) \delta 25.2,27.1,36.2,39.2,41.8$, 51.9, 60.1, 74.1, 96.0, 109.4, 110.2, 112.1, 118.9, 122.0, 125.9, 131.9, 133.4, 153.5, 174.2; HRMS (ESI): $\mathrm{m} / \mathrm{z}$ calcd for $\mathrm{C}_{19} \mathrm{H}_{20} \mathrm{Cl}_{3} \mathrm{~N}_{2} \mathrm{O}_{4}$ : $[\mathrm{M}+\mathrm{H}]^{+}$, 445.0483; found: 445.0477 .

Methyl $(6 a R, 10 a R)-4,6,6 a, 7,8,9,10,10 a-o c t a h y d r o i n d o l o-[4,3-$ fg]quinoline-9-carboxylate (7). Compound $6(20 \mathrm{~g}, 44.9 \mathrm{mmol})$ was suspended in $\mathrm{AcOH}(885 \mathrm{~mL})$ and $\mathrm{MeOH}(140 \mathrm{~mL})$ was added. Suspension was stirred vigorously at room temperature and $\mathrm{Zn}(20 \mathrm{~g}, 306 \mathrm{mmol})$ was added. After $65 \mathrm{~min} \mathrm{Zn}$ was filtered off and filtrate was concentrated under reduced pressure. To the residue on concentration $\mathrm{H}_{2} \mathrm{O}(500 \mathrm{~mL})$ was added, suspension was stirred for $0.5 \mathrm{~h}$ and filtered. Filtrate was then basified with saturated aqueous $\mathrm{NH}_{4} \mathrm{OH}$ to $\mathrm{pH} 8.1$, DCM $(400 \mathrm{~mL})$ was added 
and suspension was then acidified with glacial $\mathrm{AcOH}$ back to $\mathrm{pH}$ 6.6. Phases were separated and water phase was again basified with saturated aqueous $\mathrm{NH}_{4} \mathrm{OH}$ to $\mathrm{pH} 8.1$, DCM $(200 \mathrm{~mL})$ was added and $\mathrm{pH}$ was adjusted back to $\mathrm{pH} 6.6$ with glacial $\mathrm{AcOH}$. Phases were separated and combined organic phases were dried over $\mathrm{MgSO}_{4}$, concentrated under reduced pressure to give white solid that was suspended in heptane $(225 \mathrm{~mL})$, stirred for $1 \mathrm{~h}$ and filtered. White solid was washed with heptane and dried to give titled compound $(8.6 \mathrm{~g}, 71 \%) . R_{\mathrm{f}}: 0.40(\mathrm{AcOH}-\mathrm{heptane}=$ $1: 2) ; \mathrm{mp}=173-175{ }^{\circ} \mathrm{C}$, ref. $27172{ }^{\circ} \mathrm{C}$; $[\alpha]_{\mathrm{D}}-24$ (c 1.33; DCM); IR: $\nu_{\max } 3402,3261,3144,3093,3003,2946,2861,2801,1728$, 1601, 1548, 1517, 1435, 1248, 1234, 1199, 1109, 903, $732 \mathrm{~cm}^{-1}$; ${ }^{1} \mathrm{H} \mathrm{NMR}\left(\mathrm{CDCl}_{3}, 500 \mathrm{MHz}\right) \delta 1.63-1.72(\mathrm{~m}, 1 \mathrm{H}) ; 2.74-2.92(\mathrm{~m}$, $6 \mathrm{H})$; 2.96-3.02 (m, 1H); 3.05-3.12 (m, 1H); 3.46-3.52 (m, $1 \mathrm{H})$; $3.74(\mathrm{~s}, 3 \mathrm{H}) ; 6.88(\mathrm{~s}, 1 \mathrm{H}) ; 6.94(\mathrm{~d}, J=6.7 \mathrm{~Hz}, 1 \mathrm{H}) ; 7.15-7.22(\mathrm{~m}$, $2 \mathrm{H}) ; 8.03$ (bs, $1 \mathrm{H}) .{ }^{13} \mathrm{C} \mathrm{NMR}\left(\mathrm{CDCl}_{3}, 125 \mathrm{MHz}\right) \delta 29.6,30.8,41.3$, 43.0, 48.4, 52.0, 59.6, 109.0, 111.8, 113.3, 117.8, 123.2, 126.6, 132.5, 133.6, 174.4; HRMS (ESI): $m / z$ calcd for $\mathrm{C}_{16} \mathrm{H}_{19} \mathrm{~N}_{2} \mathrm{O}_{2}$ : $[\mathrm{M}+$ $\mathrm{H}]^{+}, 271.1441$; found: 271.1440 .

Methyl $\quad(6 a R, 9 R, 10 a R)-7-a l l y l-4,6,6 a, 7,8,9,10,10 a-o c t a h y-$ droindolo $[4,3-f g]$ quinoline-9-carboxylate (8). Compound 7 (8.00 $\mathrm{g}, 29.6 \mathrm{mmol})$ was dissolved in DMF $(58 \mathrm{~mL})$, while stirring $\mathrm{K}_{2} \mathrm{CO}_{3}(8.17 \mathrm{~g}, 59.2 \mathrm{mmol})$ and allyl bromide $(2.97 \mathrm{~mL}, 34.3$ $\mathrm{mmol})$ were added. The reaction mixture was stirred at $35^{\circ} \mathrm{C}$ for $2.5 \mathrm{~h}$. Then reaction mixture was filtered and EtOAc $(350 \mathrm{~mL})$ was added. Organic phase was washed with water $(4 \times 500 \mathrm{~mL})$ and combined water phases were reextracted with EtOAc (150 $\mathrm{mL}$ ). Combined organic phases were dried over $\mathrm{Na}_{2} \mathrm{SO}_{4}$, concentrated under reduced pressure and dried to give titled compound as a white solid (8.68 g, 94\%). $R_{\mathrm{f}}: 0.84$ (EtOAc-EtOH $=88: 12) ; \mathrm{mp}=146-149{ }^{\circ} \mathrm{C}$, ref. $10143-146{ }^{\circ} \mathrm{C} ;[\alpha]_{\mathrm{D}}-117.1(\mathrm{c}$ 1.0; DCM), ref. $10[\alpha]_{\mathrm{D}}-117.2$; IR: $\nu_{\max } 3402,3147,2948,2838$, 1729, 1641, 1610, 1547, 1443, 1365, 1201, $751 \mathrm{~cm}^{-1} ;{ }^{1} \mathrm{H}$ NMR $\left(\mathrm{CDCl}_{3}, 500 \mathrm{MHz}\right) \delta 1.63-1.73(\mathrm{~m}, 1 \mathrm{H}) ; 2.49(\mathrm{t}, J=11.6 \mathrm{~Hz} ; 1 \mathrm{H})$; $2.54\left(\mathrm{td}, J_{1}=10.1 \mathrm{~Hz}, J_{2}=4.8 \mathrm{~Hz}, 1 \mathrm{H}\right) ; 2.75(\mathrm{bt}, J=13.0 \mathrm{~Hz}, 1 \mathrm{H})$; 2.90-3.05 (m, 3H); 3.34 (d, $J=11 \mathrm{~Hz}, 1 \mathrm{H}) ; 3.38-3.49(\mathrm{~m}, 2 \mathrm{H})$; $3.60\left(\mathrm{dd}, J_{1}=8.6 \mathrm{~Hz}, J_{2}=5.8 \mathrm{~Hz}, 1 \mathrm{H}\right) ; 3.75(\mathrm{~s}, 3 \mathrm{H}) ; 5.23(\mathrm{~d}, J=$ $10.2 \mathrm{~Hz}, 1 \mathrm{H}) ; 5.29$ (d, $J=17.1 \mathrm{~Hz}, 1 \mathrm{H}) ; 5.99(\mathrm{~m}, 1 \mathrm{H}) ; 6.89(\mathrm{~s}, 1 \mathrm{H})$; $6.95(\mathrm{~d}, J=6.3 \mathrm{~Hz}, 1 \mathrm{H}) ; 7.15-7.21(\mathrm{~m}, 2 \mathrm{H}) ; 8.07$ (s, 1H). ${ }^{13} \mathrm{C} \mathrm{NMR}$ $\left(\mathrm{CDCl}_{3}, 125 \mathrm{MHz}\right) \delta 26.7,30.8,40.5,41.7,51.9,54.8,56.5,63.7$, $108.8,111.9,113.4,118.0,118.6,123.3,126.2,132.9,133.4$, 133.8, 174.7; HRMS (ESI): $m / z$ calcd for $\mathrm{C}_{19} \mathrm{H}_{23} \mathrm{~N}_{2} \mathrm{O}_{2}:[\mathrm{M}+\mathrm{H}]^{+}$, 311.1754; found: 311.1753 .

$(6 a R, 9 R, 10 a R)-7-A l l y l-4,6,6 a, 7,8,9,10,10 a-o c t a h y d r o i n d o l o[4,3-$ fg]quinoline-9-carboxylic acid A. Compound 8 (0.5 g, $1.6 \mathrm{mmol})$ was dissolved in $\mathrm{MeOH}(15 \mathrm{~mL})$ and aqueous $1 \mathrm{M} \mathrm{NaOH}$ solution ( $4 \mathrm{~mL}$ ) was added. Reaction mixture was refluxed for $45 \mathrm{~min}$. The reaction mixture was concentrated under reduced pressure and residue was dissolved in $\mathrm{H}_{2} \mathrm{O}(10 \mathrm{~mL})$. Water solution was then acidified with $\mathrm{AcOH}$ to $\mathrm{pH} 5.2$ and precipitate was stirred at $\sim 3{ }^{\circ} \mathrm{C}$ (ice/water bath) for $3 \mathrm{~h}$. Precipitate was then filtered off and washed with water to give impurity $\mathbf{A}$ as a white solid $(0.388 \mathrm{~g}$, $82 \%) \cdot R_{\mathrm{f}}: 0.58$ ( $\left.\mathrm{THF}-\mathrm{H}_{2} \mathrm{O}-\mathrm{AcOH}=98: 1: 1\right) ; \mathrm{mp}=246-247{ }^{\circ} \mathrm{C}$; $[\alpha]_{\mathrm{D}}-138.4$ (c 0.72; DMSO); IR: $\nu_{\max } 3208,2942,2322,1650,1589$, 1448, 1365, 1337, 1283, 952, $757 \mathrm{~cm}^{-1} ;{ }^{1} \mathrm{H}$ NMR (DMSO- $d_{6}, 500$ MHz) $\delta 1.31-1.41(\mathrm{~m}, 1 \mathrm{H}) ; 2.30(\mathrm{t}, J=11.5 \mathrm{~Hz}, 1 \mathrm{H}) ; 2.29-2.37(\mathrm{~m}$, $1 \mathrm{H}) ; 2.56$ (bt, $J=12.8 \mathrm{~Hz}, 1 \mathrm{H}) ; 2.66-2.75(\mathrm{~m}, 1 \mathrm{H}) ; 2.78-2.86(\mathrm{~m}$,
$2 \mathrm{H}) ; 3.16-3.21(\mathrm{~m}, 1 \mathrm{H}) ; 3.27\left(\mathrm{dd}, J_{1}=14.6 \mathrm{~Hz}, J_{2}=7.4 \mathrm{~Hz}, 1 \mathrm{H}\right)$; $3.36\left(\mathrm{dd}, J_{1}=14.6 \mathrm{~Hz}, J_{2}=3.9 \mathrm{~Hz}, 1 \mathrm{H}\right) ; 3.51\left(\mathrm{dd}, J_{1} 14.6 \mathrm{~Hz}, J_{2}=\right.$ $5.5 \mathrm{~Hz}, 1 \mathrm{H}) ; 5.17$ (d, $J=10.2 \mathrm{~Hz}, 1 \mathrm{H}) ; 5.26$ (d, $J=17.0 \mathrm{~Hz}, 1 \mathrm{H})$; 5.90-6.00 (m, 1H); $6.79(\mathrm{~d}, J=7.1 \mathrm{~Hz}, 1 \mathrm{H}) ; 6.98(\mathrm{~s}, 1 \mathrm{H}) ; 7.02(\mathrm{t}, J=$ $7.6 \mathrm{~Hz}, 1 \mathrm{H}) ; 7.14$ (d, $J=8.1 \mathrm{~Hz}, 1 \mathrm{H}) ; 10.65$ (s, $1 \mathrm{H}) .{ }^{13} \mathrm{C}$ NMR $\left(\mathrm{DMSO}-d_{6}, 125 \mathrm{MHz}\right) \delta 26.2,30.6,39.9,40.9,54.7,55.6,63.5$, 108.8, 110.1, 112.1, 117.9, 118.6, 122.1, 125.9, 132.4, 133.2, 134.7, 175.2; HRMS (ESI): $m / z$ calcd for $\mathrm{C}_{18} \mathrm{H}_{21} \mathrm{~N}_{2} \mathrm{O}_{2}$ : $[\mathrm{M}+\mathrm{H}]^{+}, 297.1598$; found: 297.1595 .

$(6 a R, 9 R, 10 a R)-7-A l l y l-N-[3-(d i m e t h y l a m i n o)$ propyl]-4,6,$6 a, 7,8,9,10,10 a$-octahydroindolo $[4,3-f g]$ quinoline-9-carboxamide D. Compound $8(0.500 \mathrm{~g}, 1.6 \mathrm{mmol})$ was dissolved in 3-dimethylamino-1-propylamine $(3 \mathrm{~mL})$ and glacial acetic acid was added $(0.1 \mathrm{~mL})$ and was stirred for $24 \mathrm{~h}$ at reflux temperature. Then the reaction mixture was cooled to room temperature and concentrated under reduced pressure to an oily brown residue. To the residue, EtOAc $(100 \mathrm{~mL})$ was added and organic phase was washed with $10 \%$ aqueous $\mathrm{NaHCO}_{3}(30 \mathrm{~mL})$ and phases were separated. Aqueous phase was reextracted with EtOAc $(2 \times 75 \mathrm{~mL})$ and combined organic phases were washed with brine $(2 \times 50 \mathrm{~mL})$. Organic phase was dried over anhydrous $\mathrm{MgSO}_{4}$, concentrated under reduced pressure and residue was crystallized from acetone to give titled compound as a light yellowish solid ( $450 \mathrm{mg}, 74 \%) \cdot R_{\mathrm{f}}: 0.18$ (EtOAc-EtOH $=$ 88 : 12); $\mathrm{mp}=201-203{ }^{\circ} \mathrm{C}$, ref. $4 a 200-202{ }^{\circ} \mathrm{C} ;[\alpha]_{\mathrm{D}}-63.0($ c 0.87; DCM); IR: $\nu_{\max } 3365,3296,3067,2941,2795,2719,1623,1545$, 1443, 1417, 1292, 911, $739 \mathrm{~cm}^{-1} ;{ }^{1} \mathrm{H} \mathrm{NMR}\left(\mathrm{CDCl}_{3}, 500 \mathrm{MHz}\right) \delta$ 1.60-1.71 (m, 3H); $2.26(\mathrm{~s}, 6 \mathrm{H}), 2.41-2.45(\mathrm{~m}, 2 \mathrm{H}) ; 2.48(\mathrm{t}, J=$ $11.5 \mathrm{~Hz}, 1 \mathrm{H}) ; 2.49-2.56(\mathrm{~m}, 1 \mathrm{H}) ; 2.62-2.68$ (m, 1H); 2.68-2.75 $(\mathrm{m}, 1 \mathrm{H}), 2.79-2.86(\mathrm{~m}, 1 \mathrm{H}) ; 2.94-3.02(\mathrm{~m}, 1 \mathrm{H}) ; 3.21-3.26(\mathrm{~m}$, $1 \mathrm{H})$; 3.36-3.43 (m, 3H); 3.46 (dd, $\left.J_{1}=14.5 \mathrm{~Hz}, J_{2}=4.1 \mathrm{~Hz}, 1 \mathrm{H}\right)$; $3.58\left(\mathrm{dd}, J_{1}=14.4 \mathrm{~Hz}, J_{2}=5.9 \mathrm{~Hz}, 1 \mathrm{H}\right) ; 5.19(\mathrm{~d}, J=10.1,1 \mathrm{H})$; $5.25\left(\mathrm{dd}, J_{1}=17.1 \mathrm{~Hz}, J_{2}=0.8 \mathrm{~Hz}, 1 \mathrm{H}\right) ; 5.92-6.03(\mathrm{~m}, 1 \mathrm{H}) ; 6.87-$ 6.93 (m, 2H); 7.13-7.20 (m, 2H); 7.64 (bs, 1H); 8.19 (s, 1H). ${ }^{13} \mathrm{C}$ NMR $\left(\mathrm{CDCl}_{3}, 125 \mathrm{MHz}\right) \delta 25.5,26.8,31.1,40.1,40.7,43.8,45.6$, 45.7, 55.8, 56.7, 59.4, 63.7, 108.8, 111.8, 113.2, 118.0, 118.7, 123.2, 126.2, 133.0, 133.4, 133.7, 173.7; HRMS (ESI): $\mathrm{m} / \mathrm{z}$ calcd for $\mathrm{C}_{23} \mathrm{H}_{33} \mathrm{~N}_{4} \mathrm{O}:[\mathrm{M}+\mathrm{H}]^{+}, 381.2649$; found: 381.2647 .

$(6 a R, 9 R, 10 a R)$-Methyl 7-allyl-4-(ethylcarbamoyl)-4,6,6a,7,8,9,10,10a-octahydroindolo[4,3-fg]quinoline-9-carboxylate

(9a).

Compound 8 (0.6 g, $1.90 \mathrm{mmol})$ in an oven dried round-bottom flask was dissolved in anhydrous THF $(16 \mathrm{~mL})$ under $\mathrm{N}_{2}$ atmosphere and cooled to $\sim 3{ }^{\circ} \mathrm{C}$ (ice/water bath). To the solution $\mathrm{NaH}$ ( $83.6 \mathrm{mg}, 60 \%$ in mineral oil, $2.1 \mathrm{mmol}$ ) was added. Mixture was stirred for additional $10 \mathrm{~min}$ and EtNCO $(0.225 \mathrm{~mL}, 2.8 \mathrm{mmol})$ was added. Reaction mixture was warmed to room temperature and stirred for $3 \mathrm{~h}$. After $3 \mathrm{~h}$ reaction mixture was cooled to $\sim 3{ }^{\circ} \mathrm{C}$ and quenched with brine $(1 \mathrm{~mL})$. Suspension was concentrated under reduced pressure and residue was suspended in DCM $(100 \mathrm{~mL})$. Water $(75 \mathrm{~mL})$ was added and phases were separated. Water phase was reextracted with ethyl acetate $(40 \mathrm{~mL})$ and combined organic phases were dried over $\mathrm{MgSO}_{4}$ and concentrated in vacuum. ${ }^{12}$ Residue on evaporation was purified using column chromatography to give title compound as a white solid $(0.341 \mathrm{~g}, 47 \%) . R_{\mathrm{f}}: 0.18$ (ethyl acetate-heptane $\left.=1: 1\right) ; \mathrm{mp}=176-$ $178{ }^{\circ} \mathrm{C} ;[\alpha]_{\mathrm{D}}-126.2$ (c 0.16; DMF); IR: $\nu_{\max } 3316,3068,3021,2977$, 2948, 2858, 2789, 1731, 1666, 1604, 1537, 1436, 1341, 1274, 1160, 
1116, 1073, 929, 752, $635 \mathrm{~cm}^{-1} ;{ }^{1} \mathrm{H}$ NMR (DMSO- $\left.d_{6}, 500 \mathrm{MHz}\right) \delta$ $1.15(\mathrm{t}, J=7.2 \mathrm{~Hz}, 1 \mathrm{H}) ; 1.32-1.42(\mathrm{~m}, 1 \mathrm{H}) ; 2.28-2.36(\mathrm{~m}, 2 \mathrm{H})$; 2.48-2.56 (m, 1H); 2.77-2.86 (m, 3H); 3.15-3.21 (m, 1H); 3.243.30 (m, 3H); 3.31-3.36 (m, 1H); 3.48-3.55 (m, 1H); 3.65 (s, 3H); $5.18(\mathrm{~d}, J=10.3 \mathrm{~Hz}, 1 \mathrm{H}) ; 5.27$ (d, $J=17.0 \mathrm{~Hz}, 1 \mathrm{H}) ; 5.87-6.00$ (m, $1 \mathrm{H}) ; 7.00$ (d, $J=7.4 \mathrm{~Hz}, 1 \mathrm{H}) ; 7.19(\mathrm{t}, J=7.7 \mathrm{~Hz}, 1 \mathrm{H}) ; 7.52(\mathrm{~d}, J=$ $1.3 \mathrm{~Hz}, 1 \mathrm{H}) ; 7.78(\mathrm{~d}, J=8.2 \mathrm{~Hz}, 1 \mathrm{H}) ; 7.99(\mathrm{t}, J=5.4 \mathrm{~Hz}, 1 \mathrm{H}) .{ }^{13} \mathrm{C}$ NMR (DMSO- $\left.d_{6}, 125 \mathrm{MHz}\right) \delta 15.0,25.7,30.3,34.9,40.6,51.6,54.3$, 55.4, 62.8, 112.9, 115.1, 115.9, 117.9, 118.1, 124.7, 127.1, 132.8, 133.1, 134.6, 151.8, 173.7; HRMS (ESI): $m / z$ calcd for $\mathrm{C}_{22} \mathrm{H}_{28} \mathrm{~N}_{3} \mathrm{O}_{3}$ : $[\mathrm{M}+\mathrm{H}]^{+}:$382.2125; 382.2118.

$(6 a R, 9 R, 10 a R)-7-A l l y l-4-(e t h y l c a r b a m o y l)-4,6,6 a, 7,8,9,10,10 a-$ octahydroindolo[4,3-fg]quinolone-9-carboxylic acid (10)

(a) Via saponification of $\mathbf{9 a}$. In a round-bottom flask compound 9a (100 mg, $0.272 \mathrm{mmol}$ ) was dissolved in $\mathrm{THF}-\mathrm{H}_{2} \mathrm{O}$ mixture $(2: 1,6 \mathrm{~mL})$. To the mixture aqueous solution of $\mathrm{NaOH}$ $(1.0 \mathrm{M}, 0.6 \mathrm{~mL})$ was added and reaction mixture was stirred for 1 h. After that aqueous $\mathrm{HCl}(1.0 \mathrm{M}, 0.9 \mathrm{~mL})$ was added reaction mixture was concentrated under reduced pressure. The residue on evaporation was purified by column chromatography (THF$\left.\mathrm{H}_{2} \mathrm{O}-\mathrm{AcOH}=98: 1: 1\right)$ to give titled compound as a yellowish solid (76.9 mg, 77\%).

(b) Via Pinnic oxidation of 15. To aldehyde 15 (1.84 g, 5.20 $\mathrm{mmol}), t$-BuOH $(18 \mathrm{~mL})$ and THF $(18 \mathrm{~mL})$ were added and solution was stirred and cooled to $\sim 3{ }^{\circ} \mathrm{C}$ (ice/water bath). Then 2-methyl-2-butene $(2.83 \mathrm{~mL}, 26.86 \mathrm{mmol})$ was added. To this a solution of $\mathrm{NaH}_{2} \mathrm{PO}_{4}(1.89 \mathrm{~g}, 15.7 \mathrm{mmol})$ and $\mathrm{NaClO}_{2}(1.42 \mathrm{~g}$, $12.6 \mathrm{mmol}, 80 \%)$ in $\mathrm{H}_{2} \mathrm{O}(18 \mathrm{~mL})$ was added slowly. Reaction mixture was stirred for $1.5 \mathrm{~h}$ at $\sim 3{ }^{\circ} \mathrm{C}$ (ice/water bath) and then it was stopped with addition of $\mathrm{Na}_{2} \mathrm{SO}_{3}(2.02 \mathrm{~g}, 16.0 \mathrm{mmol})$. Precipitate formed was filtered off and filtrate was concentrated under reduced pressure. Residue on concentration was purified by column chromatography with two different mobile phases used sequentially. First mobile phase (DCM-MeOH $=10: 1$ ) was used to separate yellow impurities and second mobile phase $\left(\mathrm{THF}-\mathrm{H}_{2} \mathrm{O}-\mathrm{AcOH}=98: 1: 1\right)$ was used to elute product which upon evaporation and drying gave light yellowish solid $(1.33 \mathrm{~g}, 68 \%) . R_{\mathrm{f}}: 0.57$ ( $\left.\mathrm{THF}-\mathrm{H}_{2} \mathrm{O}-\mathrm{AcOH}=98: 1: 1\right) ; \mathrm{mp}=155-$ $158{ }^{\circ} \mathrm{C}$ (from EtOAc); $[\alpha]_{\mathrm{D}}-73.3$ (c 1.19; DMSO); calc. for: $\mathrm{C}_{21} \mathrm{H}_{25} \mathrm{~N}_{3} \mathrm{O}_{3} \cdot \mathrm{H}_{2} \mathrm{O}: \mathrm{C}, 65.44 ; \mathrm{H}, 7.06 ; \mathrm{N}, 10.90$. Found: C, 65.03; H, 6.72; N, 10.58\%; IR: $\nu_{\max } 3308,2962,2930,2872,2854,1677$, 1586, 1532, 1439, 1390, 1274, 1035, $753 \mathrm{~cm}^{-1}$. ${ }^{1} \mathrm{H}$ NMR (DMSO$\left.d_{6}, 500 \mathrm{MHz}\right) \delta 1.15(\mathrm{t}, J=7.1 \mathrm{~Hz}, 3 \mathrm{H}) ; 1.27-1.38(\mathrm{~m}, 1 \mathrm{H}) ; 2.25-$ $2.37(\mathrm{~m}, 2 \mathrm{H}) ; 2.68(\mathrm{t}, J=10.8 \mathrm{~Hz}, 1 \mathrm{H}) ; 2.76-2.85(\mathrm{~m}, 2 \mathrm{H}) ; 3.17(\mathrm{~d}$, $J=11.1 \mathrm{~Hz}, 1 \mathrm{H}) ; 3.20-3.48(\mathrm{~m}, 5 \mathrm{H}) ; 3.52\left(\mathrm{dd}, J_{1}=14.6 \mathrm{~Hz}, J_{2}=\right.$ $3.3 \mathrm{~Hz} ; 1 \mathrm{H}) ; 5.17$ (d, $J=10.1 \mathrm{~Hz}, 1 \mathrm{H}) ; 5.27$ (d, $J=16.9 \mathrm{~Hz}, 1 \mathrm{H})$; 5.90-6.00 (m, 1H); 7.01 (d, $J=7.2 \mathrm{~Hz}, 1 \mathrm{H}) ; 7.19(\mathrm{t}, J=7.7 \mathrm{~Hz}$, $1 \mathrm{H}) ; 7.52$ (s, 1H); 7.87 (d, J=8.0 Hz, 1H); 7.99 (bs, $1 \mathrm{H}), 12.50$ (bs, $1 \mathrm{H}) .{ }^{13} \mathrm{C}$ NMR (DMSO- $\left.d_{6}, 125 \mathrm{MHz}\right) \delta 15.0,25.7,30.5,34.9,39.8$, 40.8, 54.6, 55.5, 62.9, 112.9, 115.2, 115.9, 117.8, 118.1, 124.7, 127.2, 133.0, 133.1, 134.7, 151.9, 175.1; HRMS (ESI): $\mathrm{m} / \mathrm{z}$ calcd for $\mathrm{C}_{21} \mathrm{H}_{26} \mathrm{~N}_{3} \mathrm{O}_{3}:[\mathrm{M}+\mathrm{H}]^{+}$, 368.1969; found: 368.1967.

[(6aR,9R,10aR)-7-Allyl-4,6,6a,7,8,9,10,10a-octahydroindolo[4,3-fg]quinoline-9-yl]methanol (11). In a dry round-bottom flask with compound 8 (0.200 g, $0.64 \mathrm{mmol})$, anhydrous THF $(20 \mathrm{~mL})$ was added under inert atmosphere. The solution was cooled to $\sim 3{ }^{\circ} \mathrm{C}$ (ice/water bath) and with vigorous stirring
$\mathrm{LiAlH}_{4}\left(0.97 \mathrm{~mL}, 0.97 \mathrm{mmol}, 1 \mathrm{M} / \mathrm{Et}_{2} \mathrm{O}\right)$ was added dropwise. Reaction was stopped after 45 min with addition of $\mathrm{H}_{2} \mathrm{O}$ $(0.1 \mathrm{~mL})$ and was stirred for additional $20 \mathrm{~min}$ at indicated temperature. Suspension was then filtered through celite and washed with EtOAc $(2 \times 5 \mathrm{~mL})$. Filtrate was concentrated under reduced pressure and dried in vacuum to give titled compound as white solid (157.5 mg, 87\%). $R_{\mathrm{f}}: 0.25$ (EtOAc-EtOH = $\left.88: 12\right)$; $\mathrm{mp}=206-209{ }^{\circ} \mathrm{C}$, ref. $8204-206^{\circ} \mathrm{C} ;[\alpha]_{\mathrm{D}}-74.4$ (c 0.91, DMSO); IR: $\nu_{\max } 3240,3056,2923,2847,1868,1797,1644,1612,1552$, 1469, 1445, 1342, 1035, $733 \mathrm{~cm}^{-1} ;{ }^{1} \mathrm{H}$ NMR (DMSO- $d_{6}, 500 \mathrm{MHz}$ ) $\delta$ 0.88-0.97 (m, 1H); $1.94(\mathrm{t}, J=10.9 \mathrm{~Hz}, 1 \mathrm{H}) ; 2.27\left(\mathrm{td}, J_{1}=10.2\right.$ $\left.\mathrm{Hz}, J_{2}=4.1 \mathrm{~Hz}, 1 \mathrm{H}\right) ; 2.52-2.59(\mathrm{~m}, 2 \mathrm{H}) ; 2.73-2.80(\mathrm{~m}, 1 \mathrm{H}) ; 3.07$ $(\mathrm{d}, J=9.9 \mathrm{~Hz}, 1 \mathrm{H}) ; 3.23\left(\mathrm{dd}, J_{1}=14.6 \mathrm{~Hz}, J_{2}=7.6 \mathrm{~Hz}, 1 \mathrm{H}\right) ; 3.26-$ $3.31(\mathrm{~m}, 1 \mathrm{H}) ; 3.36-3.43(\mathrm{~m}, 3 \mathrm{H}) ; 3.49\left(\mathrm{dd}, J_{1}=14.6 \mathrm{~Hz}, J_{2}=5.6\right.$ $\mathrm{Hz}, 1 \mathrm{H}) ; 4.54$ (t, $J=5.3 \mathrm{~Hz}, 1 \mathrm{H}) ; 5.15$ (d, $J=10.4 \mathrm{~Hz}, 1 \mathrm{H}) ; 5.24(\mathrm{~d}$, $J=16.8 \mathrm{~Hz}, 1 \mathrm{H}) ; 5.95(\mathrm{~m}, 1 \mathrm{H}) ; 6.77$ (d, $J=7.1 \mathrm{~Hz}, 1 \mathrm{H}) ; 6.96$ (bs, $1 \mathrm{H}) ; 7.00(\mathrm{t}, J=7.6 \mathrm{~Hz}, 1 \mathrm{H}) ; 7.11(\mathrm{~d}, J=8.1 \mathrm{~Hz}, 1 \mathrm{H}) ; 10.60(\mathrm{~s}$, $1 \mathrm{H}) .{ }^{13} \mathrm{C}$ NMR (DMSO- $\left.d_{6}, 125 \mathrm{MHz}\right) \delta 26.4,30.9,38.3,40.5,56.1$, 56.7, 64.1, 64.6, 108.6, 110.3, 112.0, 117.4, 118.4, 122.0, 126.0, 133.1, 135.1; HRMS (ESI): $m / z$ calcd for $\mathrm{C}_{18} \mathrm{H}_{23} \mathrm{~N}_{2} \mathrm{O}:[\mathrm{M}+\mathrm{H}]^{+}$, 283.1805; found: 283.1802 .

(6aR,9R,10aR)-7-Allyl-9-[(tert-butyldimethylsilyl)oxy]-methyl4,6,6a,7,8,9,10,10a-octahydroindolo[4,3- $f g]$ quinoline (12). To alcohol 11 (6.00 g, $21.2 \mathrm{mmol})$ in DMF (150 mL), imidazole (4.34 $\mathrm{g}, 63.8 \mathrm{mmol})$ and TBDMS-Cl (4.80 g, $31.9 \mathrm{mmol})$ were added. Reaction mixture was stirred for $2 \mathrm{~h}$ at room temperature after which it was quenched with saturated, aqueous solution of $\mathrm{NaHCO}_{3}(150 \mathrm{~mL})$. To a white suspension, $\mathrm{H}_{2} \mathrm{O}(500 \mathrm{~mL})$ and EtOAc $(400 \mathrm{~mL})$ were added and phases were separated after addition of brine $(30 \mathrm{~mL})$. Water phase was washed with EtOAc $(2 \times 300 \mathrm{~mL})$. Combined organic phases were reextracted with water $(3 \times 400 \mathrm{~mL})$, dried over anhydrous $\mathrm{Na}_{2} \mathrm{SO}_{4}$ and concentrated under reduced pressure. Residue was dried at $40{ }^{\circ} \mathrm{C}$ for 19 $\mathrm{h}$ to give titled compound as white solid (8.39 g, 99\%). $R_{\mathrm{f}}: 0.57$ $($ EtOAc-EtOH $=88: 12) ; \mathrm{mp}=225-227{ }^{\circ} \mathrm{C}($ from $\mathrm{MeOH}) ;[\alpha]_{\mathrm{D}}$ -69.0 (c 1.19; DCM); calc. for: $\mathrm{C}_{24} \mathrm{H}_{36} \mathrm{~N}_{2} \mathrm{OSi}$ : C, 72.67; H, 9.15; N, 7.06. Found: C, 72.73; H, 9.39; N, 7.10\%; IR: $\nu_{\max } 3096,3013$, 2949, 2929, 2899, 2856, 2813, 2784, 1738, 1645, 1618, 1549, 1518, 1462, 1357, 1217, 1098, 834, 772, $742 \mathrm{~cm}^{-1} ;{ }^{1} \mathrm{H}$ NMR $\left(\mathrm{CDCl}_{3}, 500 \mathrm{MHz}\right) \delta 0.08(\mathrm{~s}, 3 \mathrm{H}) ; 0.09(\mathrm{~s}, 3 \mathrm{H}) ; 0.93(\mathrm{~s}, 9 \mathrm{H}) ; 1.09-$ $1.18(\mathrm{~m}, 1 \mathrm{H}) ; 2.02-2.16(\mathrm{~m}, 2 \mathrm{H}) ; 2.49\left(\mathrm{dt}, J_{1}=3.8 \mathrm{~Hz}, J_{2}=10.9\right.$ $\mathrm{Hz}, 1 \mathrm{H}) ; 2.66$ (d, $J=13.0 \mathrm{~Hz}, 1 \mathrm{H}) ; 2.75(\mathrm{t}, J=12.7 \mathrm{~Hz}, 1 \mathrm{H}) ; 3.00$ (bt, $J=9.1 \mathrm{~Hz}, 1 \mathrm{H}) ; 3.21(\mathrm{~d}, J=9.5 \mathrm{~Hz}, 1 \mathrm{H}) ; 3.38\left(\mathrm{dd}, J_{1}=14.2\right.$ $\left.\mathrm{Hz}, J_{2}=7.5 \mathrm{~Hz}, 1 \mathrm{H}\right) ; 3.47\left(\mathrm{dd}, J_{1}=14.6 \mathrm{~Hz}, J_{2}=3.9 \mathrm{~Hz}, 1 \mathrm{H}\right)$; $3.51-3.56(\mathrm{~m}, 1 \mathrm{H}) ; 3.58-3.63(\mathrm{~m}, 2 \mathrm{H}) ; 5.20$ (d, $J=10.1 \mathrm{~Hz}, 1 \mathrm{H})$; $5.26(\mathrm{~d}, J=17.0 \mathrm{~Hz}, 1 \mathrm{H}) ; 5.95-6.06(\mathrm{~m}, 1 \mathrm{H}) ; 6.89$ (s, 1H); 6.92$6.95(\mathrm{~m}, 1 \mathrm{H}) ; 7.15-7.20(\mathrm{~m}, 2 \mathrm{H}), 8.01(\mathrm{~s}, 1 \mathrm{H}) .{ }^{13} \mathrm{C} \mathrm{NMR}\left(\mathrm{CDCl}_{3}\right.$, $125 \mathrm{MHz}) \delta-5.2,-5.1,1.2,18.5,26.1,26.8,29.8,31.0,38.7$, 40.9, 56.8, 56.9, 64.3, 66.7, 108.58, 112.3, 113.3, 117.8, 118.2, 123.2, 126.4, 133.4, 134.0, 134.1; HRMS (ESI): $\mathrm{m} / \mathrm{z}$ calcd for $\mathrm{C}_{24} \mathrm{H}_{37} \mathrm{~N}_{2} \mathrm{OSi}:[\mathrm{M}+\mathrm{H}]^{+}$, 397.2675; found: 397.2673 .

(6aR,9R,10aR)-7-Allyl-9-[(tert-butyldimethylsilyl)oxy]-methyl$N$-ethyl-6a,7,8,9,10,10a-hexahydroindolo[4,3-fg]quinoline-4(6H)carboxamide (13). Compound $12(16.00 \mathrm{~g}, 40.4 \mathrm{mmol})$ in an oven dried glassware was dissolved in anhydrous THF $(400 \mathrm{~mL})$ under $\mathrm{N}_{2}$ atmosphere. Solution was cooled to $\sim 3{ }^{\circ} \mathrm{C}$ (ice/water bath) and $\mathrm{NaH}$ (1.86 g, 60\% in mineral oil, $44.4 \mathrm{mmol}$ ) was added. 
Suspension was stirred for additional $10 \mathrm{~min}$ at indicated temperature and EtNCO $(4.80 \mathrm{~mL}, 60.6 \mathrm{mmol})$ was added. Reaction mixture was then warmed to room temperature and stirred for $3 \mathrm{~h}$. After $3 \mathrm{~h}$ reaction mixture was cooled to $\sim 3{ }^{\circ} \mathrm{C}$ and quenched with brine $(20.4 \mathrm{~mL})$. Suspension was concentrated under reduced pressure, oily residue was dissolved in DCM $(1000 \mathrm{~mL})$ and washed with $\mathrm{H}_{2} \mathrm{O}(400 \mathrm{~mL})$. Water phase was then reextracted with DCM $(2 \times 250 \mathrm{~mL})$ and, combined organic phases were additionally washed with water $(200 \mathrm{~mL})$. Organic phase was dried over anhydrous $\mathrm{Na}_{2} \mathrm{SO}_{4}$ and concentrated under reduced pressure to give brown solid, which was purified by column chromatography (EtOAc-heptane $=1: 3$ ) to give titled compound as pale yellow solid $(16.94 \mathrm{~g}, 90 \%) . R_{\mathrm{f}}: 0.38$ (EtOAcheptane $=1: 3) ; \mathrm{mp}=135-137{ }^{\circ} \mathrm{C}\left(\right.$ from $\left.\mathrm{MeOH} / \mathrm{H}_{2} \mathrm{O}\right) ;[\alpha]_{\mathrm{D}}$ -75.9 (c 1.40; DCM); calc. for: $\mathrm{C}_{27} \mathrm{H}_{41} \mathrm{~N}_{3} \mathrm{O}_{2} \mathrm{Si}$ : C, 69.33; H, 8.84; N, 8.98. Found: C, 69.58; H, 9.11; N, 9.02\%; IR: $\nu_{\max } 3377,2952$, 2925, 2853, 2810, 1737, 1661, 1605, 1535, 1460, 1449, 1438, $1291,1087,837,780,754 \mathrm{~cm}^{-1} ;{ }^{1} \mathrm{H} \mathrm{NMR}\left(\mathrm{CDCl}_{3}, 500 \mathrm{MHz}\right) \delta 0.07$ $(\mathrm{s}, 3 \mathrm{H}) ; 0.08(\mathrm{~s}, 3 \mathrm{H}) ; 0.91(\mathrm{~s}, 9 \mathrm{H}) ; 1.05-1.13(\mathrm{~m}, 1 \mathrm{H}) ; 1.28(\mathrm{t}, J=7.2$ $\mathrm{Hz}, 3 \mathrm{H}) ; 2.00-2.13(\mathrm{~m}, 2 \mathrm{H}), 2.40\left(\mathrm{td}, J_{1}=11.2 \mathrm{~Hz}, J_{2}=3.9 \mathrm{~Hz}\right.$, $1 \mathrm{H}) ; 2.58-2.63(\mathrm{~m}, 2 \mathrm{H}) ; 2.91$ (bt, $J=9.1 \mathrm{~Hz}, 1 \mathrm{H}) ; 3.18$ (d, $J=8.6$ $\mathrm{Hz}, 1 \mathrm{H}) ; 3.31\left(\mathrm{dd}, J_{1}=14.5 \mathrm{~Hz}, J_{2}=7.6 \mathrm{~Hz}, 1 \mathrm{H}\right) ; 3.36\left(\mathrm{dd}, J_{1}=\right.$ $\left.15.0 \mathrm{~Hz}, J_{2}=3.9 \mathrm{~Hz}, 1 \mathrm{H}\right) ; 3.46-3.64(\mathrm{~m}, 5 \mathrm{H}) ; 5.19(\mathrm{~d}, J=10.2 \mathrm{~Hz}$, $1 \mathrm{H}) ; 5.24$ (d, $J=17.1 \mathrm{~Hz}, 1 \mathrm{H}) ; 5.66$ (bs, $1 \mathrm{H}) ; 5.90-6.01(\mathrm{~m}, 1 \mathrm{H})$; $7.04(\mathrm{~d}, J=7.3 \mathrm{~Hz}, 1 \mathrm{H}) ; 7.20$ (s, 1H); 7.27 (t, $J=7.8 \mathrm{~Hz}, 1 \mathrm{H}) ; 7.72$ $(\mathrm{d}, J=8.2 \mathrm{~Hz}, 1 \mathrm{H}) .{ }^{13} \mathrm{C} \mathrm{NMR}\left(\mathrm{CDCl}_{3}, 125 \mathrm{MHz}\right) \delta-5.23,-5.19$, 15.1, 15.3, 18.4, 26.1, 26.5, 29.8, 30.9, 35.9, 38.5, 40.5, 56.6, 56.7, 63.8, 66.6, 111.7, 116.4, 116.5, 117.9, 118.4, 125.3, 128.4, 132.8, 133.8, 134.4, 152.5; HRMS (ESI): $m / z$ calcd for $\mathrm{C}_{27} \mathrm{H}_{42} \mathrm{~N}_{3} \mathrm{O}_{2} \mathrm{Si}$ : [M $+\mathrm{H}]^{+}$, 468.3041; found: 468.3040 .

$(6 a R, 9 R, 10 a R)-7-A l l y l-N$-ethyl-9-(hydroxymethyl)-6a,7,8,-9,10,10ahexahydroindolo $[4,3-f g]$ quinoline-4(6H)-carboxamide

(14).

Compound 13 (4.5 g, $9.6 \mathrm{mmol})$ was dissolved in THF (50 mL). Solution was cooled to $0{ }^{\circ} \mathrm{C}$, TBAF $(19.2 \mathrm{~mL}, 19.2 \mathrm{mmol}, 1 \mathrm{M}$ in THF) was added and reaction mixture was stirred at indicated temperature for $45 \mathrm{~h}$. After that $\mathrm{H}_{2} \mathrm{O}(0.38 \mathrm{~mL})$ was added and reaction mixture was concentrated under reduced pressure. Residue was purified by column chromatography $(\mathrm{EtOH}-\mathrm{EtOAc}=12: 88)$, to give titled compound as a white solid $(3.21 \mathrm{~g}, 94 \%) . R_{\mathrm{f}}: 0.38$ (EtOHEtOAc $=12: 88) ; \mathrm{mp}=190-193{ }^{\circ} \mathrm{C}$ (from toluene); $[\alpha]_{\mathrm{D}}-92.5(c$ 1.26; DMSO); calc. for: $\mathrm{C}_{21} \mathrm{H}_{27} \mathrm{~N}_{3} \mathrm{O}_{2}$ : C, 71.36; $\mathrm{H}, 7.70 ; \mathrm{N}, 11.89$. Found: C, 71.65; H, 8.02; N, 11.89\%; IR: $\nu_{\max } 3424,3303,2932,2913$, 2856, 2812, 2784, 1664, 1603, 1533, 1439, 1243, 1142, 1039, 918, 812, $748 \mathrm{~cm}^{-1} ;{ }^{1} \mathrm{H}$ NMR $\left(\mathrm{CDCl}_{3}, 500 \mathrm{MHz}\right) \delta 1.08-1.18(\mathrm{~m}, 1 \mathrm{H}) ; 1.30(\mathrm{t}$, $J=7.2 \mathrm{~Hz}, 3 \mathrm{H}) ; 1.80$ (bs, $1 \mathrm{H}) ; 2.08-2.17$ (m, 2H); 2.44 (td, $J_{1}=10.5$ $\left.\mathrm{Hz}, J_{2}=4.0 \mathrm{~Hz}, 1 \mathrm{H}\right) ; 2.63-2.71(\mathrm{~m}, 2 \mathrm{H}) ; 2.92-2.93(\mathrm{~m}, 1 \mathrm{H}) ; 3.16-3.24$ (m, 1H); 3.34-3.43 (m, 2H); 3.49-3.55 (m, 2H); 3.55-3.62 (m, 2H); $3.66\left(\mathrm{dd}, J_{1}=10.8 \mathrm{~Hz}, J_{2}=4.7 \mathrm{~Hz}, 1 \mathrm{H}\right) ; 5.21(\mathrm{~d}, J=10.2 \mathrm{~Hz}, 1 \mathrm{H}) ; 5.26$ $(\mathrm{d}, J=16.8 \mathrm{~Hz}, 1 \mathrm{H}) ; 5.50(\mathrm{t}, J=4.7 \mathrm{~Hz}, 1 \mathrm{H}) ; 5.92-6.02(\mathrm{~m}, 1 \mathrm{H}) ; 7.06$ $(\mathrm{d}, J=7.4 \mathrm{~Hz}, 1 \mathrm{H}) ; 7.20$ (d, $J=1.3 \mathrm{~Hz}, 1 \mathrm{H}) ; 7.28(\mathrm{t}, J=8.0 \mathrm{~Hz}, 1 \mathrm{H})$; $7.69(\mathrm{~d}, J=8.2 \mathrm{~Hz}, 1 \mathrm{H}) ;{ }^{1} \mathrm{H}$ NMR (DMSO- $\left.d_{6}, 500 \mathrm{MHz}\right) \delta 0.85-0.95$ $(\mathrm{m}, 1 \mathrm{H}) ; 1.15(\mathrm{t}, J=7.2 \mathrm{~Hz}, 3 \mathrm{H}) ; 1.95\left(\mathrm{dd}, J_{1}=22.3 \mathrm{~Hz}, J_{2}=11.2 \mathrm{~Hz}\right.$, $1 \mathrm{H}) ; 2.25\left(\mathrm{dt}, J_{1}=10.3 \mathrm{~Hz}, J_{2}=3.8 \mathrm{~Hz}, 1 \mathrm{H}\right) ; 2.46-2.53(\mathrm{~m}, 1 \mathrm{H}), 2.56$ (bd, $J=12.7 \mathrm{~Hz}, 1 \mathrm{H}) ; 2.71-2.79(\mathrm{~m}, 1 \mathrm{H}) ; 3.07$ (d, $J=10.2 \mathrm{~Hz}, 1 \mathrm{H})$; $3.22\left(\mathrm{dd}, J_{1}=14.6 \mathrm{~Hz}, J_{2}=7.7 \mathrm{~Hz} ; 1 \mathrm{H}\right) ; 3.25-3.32(\mathrm{~m}, 3 \mathrm{H}) ; 3.32-3.42$ $(\mathrm{m}, 3 \mathrm{H}) ; 3.50\left(\mathrm{dd}, J_{1}=14.6 \mathrm{~Hz}, J_{2}=5.3 \mathrm{~Hz} ; 1 \mathrm{H}\right) ; 4.57(\mathrm{t}, J=5.1 \mathrm{~Hz}$, $1 \mathrm{H}) ; 5.16(\mathrm{~d}, J=10.3 \mathrm{~Hz}, 1 \mathrm{H}) ; 5.24(\mathrm{~d}, J=17.1 \mathrm{~Hz}, 1 \mathrm{H}) ; 5.89-5.99(\mathrm{~m}$,
$1 \mathrm{H}) ; 6.98(\mathrm{~d}, J=7.3 \mathrm{~Hz}, 1 \mathrm{H}) ; 7.18(\mathrm{t}, J=7.8 \mathrm{~Hz}, 1 \mathrm{H}) ; 7.50$ (s, 1H); 7.85 $(\mathrm{d}, J=8.2 \mathrm{~Hz}, 1 \mathrm{H}) ; 7.97$ (t, $J=5.4 \mathrm{~Hz}, 1 \mathrm{H}) .{ }^{13} \mathrm{C} \mathrm{NMR}\left(\mathrm{CDCl}_{3}, 125\right.$ MHz) $\delta 15.3,26.5$, 30.9, 36.0, 38.6, 40.5, 56.4, 56.8, 63.7, 66.5, 111.7, 116.3, 116.6, 118.4, 118.8, 125.4, 128.4, 132.7, 133.5, 134.2, $152.4 ;{ }^{13} \mathrm{C}$ NMR (DMSO- $\left.d_{6}, 125 \mathrm{MHz}\right) \delta 15.0,25.9,30.8,34.9,38.2,40.1,56.1$, 56.5, 63.6, 64.6, 112.7, 115.4, 115.9, 117.6, 118.0, 124.7, 127.3, 133.1, 133.6, 134.9, 151.9; HRMS (ESI): $m / z$ calcd for $\mathrm{C}_{21} \mathrm{H}_{28} \mathrm{~N}_{3} \mathrm{O}_{2}:[\mathrm{M}+\mathrm{H}]^{+}$, 354.2176; found: 354.2177 .

$(6 a R, 9 R, 10 a R)$-7-Allyl- $N$-ethyl-9-formyl-6a,7,8,9,10,10a-hexahydroindolo[4,3-fg]quinoline-4(6H)-carboxamide (15) $\cdot^{17,18}$

(a) Via Pfitzner-Moffatt oxidation of 14. Alcohol 14 (3.00 g, $8.49 \mathrm{mmol}$ ) was dissolved in anhydrous DMSO $(20 \mathrm{~mL})$ and anhydrous DCM $(20 \mathrm{~mL})$ under $\mathrm{N}_{2}$ atmosphere. Solution was cooled to $\sim 3{ }^{\circ} \mathrm{C}$ (ice/water bath) and with stirring DCC (5.26 g, $25.5 \mathrm{mmol})$, anhydrous pyridine $(1.38 \mathrm{~mL}, 17 \mathrm{mmol})$ and TFA (0.99 $\mathrm{mL}, 12.7 \mathrm{mmol}$ ) were added sequentially. Reaction mixture was stirred for $1.5 \mathrm{~h}$ at $\sim 3{ }^{\circ} \mathrm{C}$ (ice/water bath). Suspension formed was filtered and to the filtrate DCM (250 $\mathrm{mL})$ was added and organic phase was washed with water $(3 \times$ $250 \mathrm{~mL})$. Combined water phases were reextracted with DCM (250 $\mathrm{mL})$. Then combined organic phases were dried over anhydrous $\mathrm{Na}_{2} \mathrm{SO}_{4}$, concentrated under reduced pressure and residue was purified by column chromatography $(\mathrm{MeOH}-\mathrm{EtOAc}$ $=10: 90$ and DCM-MeOH $=90: 10$ ) to give titled aldehyde as a white solid (2.60 g, 87\%).

(b) Via Parikh-Doering oxidation of 14. In an oven dried, round-bottom flask, alcohol 14 (50 $\mathrm{mg}, 0.141 \mathrm{mmol}$ ) under $\mathrm{N}_{2}$ atmosphere, was dissolved in anhydrous DMSO $(2 \mathrm{~mL})$ and to the solution anhydrous DCM $(2 \mathrm{~mL})$ was added. $\mathrm{Et}_{3} \mathrm{~N}(0.197 \mathrm{~mL}$, $1.41 \mathrm{mmol}$ ) was added and reaction mixture was cooled to $\sim 3{ }^{\circ} \mathrm{C}$ (ice/water bath). To this solution $\mathrm{SO}_{3} \cdot \mathrm{Py}(67.4 \mathrm{mg}, 0.423 \mathrm{mmol})$ dissolved in anhydrous DMSO $(0.25 \mathrm{~mL})$ was added dropwise and reaction mixture was stirred for $80 \mathrm{~min}$. After that time reaction mixture was poured in to $\mathrm{H}_{2} \mathrm{O}(20 \mathrm{~mL})$ and water phase was extracted with DCM $(3 \times 15 \mathrm{~mL})$. Combined organic phases were dried over $\mathrm{MgSO}_{4}$ and concentrated under reduced pressure. Residue on concentration was purified by column chromatography (EtOH-EtOAc $=1: 9)$ to give aldehyde 15 as a white solid (33.7 mg, 68\%). $R_{\mathrm{f}}: 0.56(\mathrm{MeOH}-\mathrm{EtOAc}=10: 90) ; \mathrm{mp}=$ $165-167{ }^{\circ} \mathrm{C}$ (from DCM/heptane); $[\alpha]_{\mathrm{D}}-70.0$ (c 0.45; DCM); calc. for: $\mathrm{C}_{21} \mathrm{H}_{25} \mathrm{~N}_{3} \mathrm{O}_{2}$ : C, 71.77; $\mathrm{H}, 7.17 ; \mathrm{N}, 11.96$. Found: $\mathrm{C}, 71.61 ; \mathrm{H}$, 7.31; N, 11.84\%; IR: $\nu_{\max } 3320,2927,2849,1726,1668,1623$, 1566, 1535, 1437, 1242, 1085, 892, 752, $640 \mathrm{~cm}^{-1} ;{ }^{1} \mathrm{H}$ NMR $\left(\mathrm{CDCl}_{3}, 300 \mathrm{MHz}\right) \delta 1.30(\mathrm{t}, J=7.2 \mathrm{~Hz}, 3 \mathrm{H}) ; 1.35-1.45(\mathrm{~m}, 1 \mathrm{H})$; $2.31(\mathrm{t}, J=11.6 \mathrm{~Hz}, 1 \mathrm{H}) ; 2.42-2.50(\mathrm{~m}, 1 \mathrm{H}) ; 2.65-2.75(\mathrm{~m}, 1 \mathrm{H})$; 2.85-3.05 (m, 3H); 3.30-3.44 (m, 3H); 3.45-3.63 (m, 3H); 5.19$5.32(\mathrm{~m}, 2 \mathrm{H}) ; 5.59$ (bt, $J=5.0 \mathrm{~Hz}, 1 \mathrm{H}) ; 5.90-6.00(\mathrm{~m}, 1 \mathrm{H}) ; 7.07$ (d, $J=7.4 \mathrm{~Hz}, 1 \mathrm{H}) ; 7.21(\mathrm{~d}, J=1.6 \mathrm{~Hz}, 1 \mathrm{H}) ; 7.30(\mathrm{t}, J=7.6 \mathrm{~Hz}, 1 \mathrm{H})$; $7.72(\mathrm{~d}, J=8.3 \mathrm{~Hz}, 1 \mathrm{H}) ; 9.76(\mathrm{~s}, 1 \mathrm{H}) .{ }^{13} \mathrm{C} \mathrm{NMR}\left(\mathrm{CDCl}_{3}, 75.5 \mathrm{MHz}\right)$ $\delta 15.3,26.4,27.9,36.0,40.2,48.6,52.5,56.5,63.5,112.0,115.9$, $116.5,118.2$, 118.9, 125.4, 128.3, 132.8, 133.3, 133.4, 152.4, 202.6; HRMS (ESI): $m / z$ calcd for $\mathrm{C}_{21} \mathrm{H}_{26} \mathrm{~N}_{3} \mathrm{O}_{2}:[\mathrm{M}+\mathrm{H}]^{+}$, 352.2020; found: 352.2019 .

$(6 a R, 9 R, 10 a R)-7-A l l y l-N^{9}$-[3-(dimethylamino)propyl]- $N^{4}$-ethyl$6 a, 7,8,9,10,10 a$-hexahydroindolo[4,3-fg]quinoline-4,9-(6H)-dicarboxamide B. Carboxylic acid $10(0.580 \mathrm{~g}, 1.58 \mathrm{mmol})$ in oven dried glassware was dissolved in anhydrous DMF $(10 \mathrm{~mL})$ under 
$\mathrm{N}_{2}$ atmosphere and CDI (0.267 g, $\left.1.74 \mathrm{mmol}\right)$ was added. Mixture was stirred at room temperature for $3.5 \mathrm{~h}$ and then 3-dimethylamino-1-propylamine $(0.221 \mathrm{~mL}, 1.74 \mathrm{mmol})$ was added. Reaction mixture was stirred at room temperature for $20 \mathrm{~h}$. After indicated time, $\mathrm{H}_{2} \mathrm{O}(100 \mathrm{~mL})$ was added and reaction mixture was extracted with DCM $(4 \times 75 \mathrm{~mL})$. Combined organic fractions were washed with $\mathrm{H}_{2} \mathrm{O}(3 \times 100 \mathrm{~mL})$ and concentrated under reduced pressure. Residue on evaporation was suspended in $\mathrm{H}_{2} \mathrm{O}(75 \mathrm{~mL})$. Suspension was cooled to $\sim 3{ }^{\circ} \mathrm{C}$ (ice/water bath) and acidified with $1 \mathrm{M}$ aqueous $\mathrm{HCl}$ to $\mathrm{pH} 3.75$ to obtain solution which was filtered and extracted with DCM $(2 \times 75 \mathrm{~mL})$. Organic phases were discarded and water phase was re-cooled to $\sim 3{ }^{\circ} \mathrm{C}$ (ice/water bath), basified with $1 \mathrm{M}$ aqueous $\mathrm{NaOH}$ to $\mathrm{pH} 9.60$ and precipitate formed was filtered off and washed with $\mathrm{H}_{2} \mathrm{O}(20 \mathrm{~mL})$. Solid residue on filtration was dried to give titled compound as a pale yellowish solid $(0.385 \mathrm{~g}, 54 \%)$. $R_{\mathrm{f}}: 0.25$ (EtOAc-BuOH-pyr.$\mathrm{DMF}=40: 25: 10: 25) ; \mathrm{mp}=205-207{ }^{\circ} \mathrm{C} ;[\alpha]_{\mathrm{D}}-75.4(c \quad 0.83$; DCM); IR: $\nu_{\max } 3294,3066,2934,2861,2765,2725,1663,1639$, 1535, 1439, 1346, 1212, 919, $751 \mathrm{~cm}^{-1} ;{ }^{1} \mathrm{H} \mathrm{NMR}\left(\mathrm{CDCl}_{3}, 500\right.$ MHz) $\delta 1.28(\mathrm{t}, J=7.2 \mathrm{~Hz}, 3 \mathrm{H}) ; 1.57(\mathrm{~m}, 1 \mathrm{H}) ; 1.64-1.71(\mathrm{~m}, 2 \mathrm{H})$; $2.26(\mathrm{~s}, 6 \mathrm{H}) ; 2.35-2.46(\mathrm{~m}, 4 \mathrm{H}) ; 2.51-2.58(\mathrm{~m}, 1 \mathrm{H}) ; 2.58-2.64(\mathrm{~m}$, $1 \mathrm{H}) ; 2.72-2.78(\mathrm{~m}, 1 \mathrm{H}) ; 2.79-2.86(\mathrm{~m}, 1 \mathrm{H}) ; 3.15-3.20(\mathrm{~m}, 1 \mathrm{H}) ; 3.27$ $\left(\mathrm{dd}, J_{1}=14.4 \mathrm{~Hz}, J_{2}=7.7 \mathrm{~Hz}, 1 \mathrm{H}\right) ; 3.33\left(\mathrm{dd}, J_{1}=14.9 \mathrm{~Hz}, J_{2}=4.0\right.$ $\mathrm{Hz}, 1 \mathrm{H}) ; 3.36-3.40$ (m, 2H); 3.46-3.54 (m, 3H); 5.17 (d, $J=10.2$ $\mathrm{Hz}, 1 \mathrm{H}) ; 5.22$ (d, $J=17.1 \mathrm{~Hz}, 1 \mathrm{H}) ; 5.79$ (t, $J=5.4 \mathrm{~Hz}, 1 \mathrm{H}) ; 5.86-$ $5.96(\mathrm{~m}, 1 \mathrm{H}) ; 7.00(\mathrm{~d}, J=7.3 \mathrm{~Hz}, 1 \mathrm{H}) ; 7.22(\mathrm{bs}, 1 \mathrm{H}) ; 7.23-7.27(\mathrm{~m}$, $1 \mathrm{H}) ; 7.69$ (bt, $J=4.6 \mathrm{~Hz}, 1 \mathrm{H}) ; 7.71$ (d, $J=8.2 \mathrm{~Hz}, 1 \mathrm{H}) .{ }^{13} \mathrm{C} \mathrm{NMR}$ $\left(\mathrm{CDCl}_{3}, 125 \mathrm{MHz}\right) \delta 15.3,25.5,26.4,31.0,35.9,40.0,40.3,43.7$, 45.5, 55.6, 56.5, 59.3, 63.1, 111.9, 116.0, 116.4, 118.1, 118.7, 125.3, 128.2, 132.8, 133.6, 152.5, 173.6; HRMS (ESI): $\mathrm{m} / \mathrm{z}$ calcd for $\mathrm{C}_{26} \mathrm{H}_{38} \mathrm{~N}_{5} \mathrm{O}_{2}:[\mathrm{M}+\mathrm{H}]^{+}$, 452.3020; found: 452.3019 .

$(6 a R, 9 R, 10 a R)-7-A l l y l-N^{9}$-[3-(dimethylamino)propyl]- $N^{4}$-ethyl$N^{9}$-(ethylcarbamoyl)-6a,7,8,9,10,10a[hexahydroindolo-[4,3-fg]quinoline-4,9(6H)-dicarboxamide (C) and regioisomer $(6 a R, 9 R, 10 a R)-7-a l l y l-N^{9}$-[(3-(dimethylamino)propyl)carbamoyl]$N^{4}, N^{9}$-diethyl-6a,7,8,9,10,10a-hexahydroindolo[4,3- $\left.f g\right]$ quinoline4,9(6H)-dicarboxamide (16). Carboxylic acid 10 (0.490 g, 1.33 $\mathrm{mmol}$ ) in an oven dried glassware, under $\mathrm{N}_{2}$ atmosphere was suspended in anhydrous DCM ( $8 \mathrm{~mL})$. TEA (0.37 mL, $2.66 \mathrm{mmol})$ and $\mathrm{EDC} \cdot \mathrm{HCl}$ were added and reaction mixture was stirred for $23 \mathrm{~h}$ at room temperature. After that reaction was stopped with addition of $\mathrm{H}_{2} \mathrm{O}(30 \mathrm{~mL})$ and solution was extracted with DCM (2 $\times 30 \mathrm{~mL}$ ). Combined organic phases were concentrated under reduced pressure and residue was purified by column chromatography (pyr.- $\mathrm{Me}_{2} \mathrm{CO}=10: 90$ ). First the desired product, impurity $\mathbf{C}$, was eluted and evaporation of mobile phase gave titled compound as light yellowish foam (0.405 g, 58\%). Part of the foam $(55 \mathrm{mg})$ was further crystallized from toluene $(0.2 \mathrm{~mL})$ and heptane $(3 \mathrm{~mL})$ at $-40{ }^{\circ} \mathrm{C}$ to give light yellowish solid (45 mg, 82\%). $R_{\mathrm{f}}: 0.46$ (Pyr.- $\mathrm{Me}_{2} \mathrm{CO}=10 \%: 90 \%$ ); $\mathrm{mp}=109-$ $110{ }^{\circ} \mathrm{C}$, ref. $4 a 125-127{ }^{\circ} \mathrm{C}$ (from diisopropyl ether); $[\alpha]_{\mathrm{D}}-71.8$ (c 0.19; DCM); IR: $\nu_{\max } 3334,3274,2971,2971,2936,2867,2828$, 2781, 2245, 1668, 1525, 1438, 1340, 1525, 1438, 1340, 1271, 1138, 913, $729 \mathrm{~cm}^{-1} ;{ }^{1} \mathrm{H} \mathrm{NMR}\left(\mathrm{CDCl}_{3}, 500 \mathrm{MHz}\right) \delta 1.18(\mathrm{t}, J=7.2 \mathrm{~Hz}$, $3 \mathrm{H}) ; 1.29(\mathrm{t}, J=7.2 \mathrm{~Hz}, 3 \mathrm{H}) ; 1.73(\mathrm{~m}, 1 \mathrm{H}) ; 1.83-1.91(\mathrm{~m}, 2 \mathrm{H}) ; 2.28$ $(\mathrm{s}, 6 \mathrm{H}) ; 2.34-2.44(\mathrm{~m}, 2 \mathrm{H}) ; 2.49-2.58(\mathrm{~m}, 2 \mathrm{H}) ; 2.63(\mathrm{t}, J=12.9 \mathrm{~Hz}$, $1 \mathrm{H}) ; 2.79$ (d, $J=11.8 \mathrm{~Hz}, 1 \mathrm{H}) ; 2.95$ (bt, $J=8.9 \mathrm{~Hz}, 1 \mathrm{H}) ; 3.16$ $(\mathrm{d}, J=10.8 \mathrm{~Hz}, 1 \mathrm{H}) ; 3.26-3.40(\mathrm{~m}, 5 \mathrm{H}) ; 3.47-3.58(\mathrm{~m}, 3 \mathrm{H}) ; 3.72-$ $3.90(\mathrm{~m}, 2 \mathrm{H}) ; 5.19$ (d, $J=10.0 \mathrm{~Hz}, 1 \mathrm{H}) ; 5.25$ (d, $J=17.1 \mathrm{~Hz}, 1 \mathrm{H})$; 5.62 (bt, $J=5.3 \mathrm{~Hz}, 1 \mathrm{H}) ; 5.88-5.99(\mathrm{~m}, 1 \mathrm{H}), 7.02(\mathrm{~d}, J=7.2 \mathrm{~Hz}$, $1 \mathrm{H}) ; 7.26$ (t, $J=7.7 \mathrm{~Hz}, 1 \mathrm{H}), 7.70(\mathrm{~d}, J=8.2 \mathrm{~Hz}, 1 \mathrm{H}) ; 9.45$ (bs, $1 \mathrm{H})$. ${ }^{13} \mathrm{C} \mathrm{NMR}\left(\mathrm{CDCl}_{3}, 125 \mathrm{MHz}\right) \delta 14.9,15.3,26.5,29.8,30.4,31.3$, 35.6, 36.0, 39.9, 42.3, 43.3, 44.9, 55.7, 56.2, 63.4, 111.8, 116.1, $116.6,118.1,118.7,125.4,128.3,132.7,133.5,133.7,152.4$, 177.6; HRMS (ESI): $m / z$ calcd for $\mathrm{C}_{29} \mathrm{H}_{43} \mathrm{~N}_{6} \mathrm{O}_{3}:[\mathrm{M}+\mathrm{H}]^{+}, 523.3391$; found: 523.3390 .

Continuing elution, regioisomer 16 was isolated and evaporation of mobile phase gave $(67 \mathrm{mg}, 10 \%)$ yellow oil. $R_{\mathrm{f}}: 0.29$ (pyr. $\left.-\mathrm{Me}_{2} \mathrm{CO}=10: 90\right) ;[\alpha]_{\mathrm{D}}-40.2$ (c 0.10; DCM); IR: $\nu_{\max } 3282$, 2971, 2935, 2868, 2817, 2785, 2246, 1688, 1524, 1438, 1340, 1271, 1139, 917, $729 \mathrm{~cm}^{-1} ;{ }^{1} \mathrm{H}$ NMR $\left(\mathrm{CDCl}_{3}, 500 \mathrm{MHz}\right) \delta 1.28(\mathrm{t}$, $J=7.2 \mathrm{~Hz}, 3 \mathrm{H}) ; 1.30(\mathrm{t}, J=6.7 \mathrm{~Hz}, 3 \mathrm{H}) ; 1.72-1.83(\mathrm{~m}, 3 \mathrm{H}) ; 2.27$ $(\mathrm{s}, 6 \mathrm{H}) ; 2.40(\mathrm{~m}, 2 \mathrm{H}) ; 2.50-2.66(\mathrm{~m}, 3 \mathrm{H}) ; 2.74(\mathrm{~d}, J=13.0 \mathrm{~Hz}, 1 \mathrm{H})$; 2.92-2.99 (m, 1H); 3.15 (d, $J=13.4 \mathrm{~Hz}, 1 \mathrm{H}) ; 3.21$ (d, $J=11.4 \mathrm{~Hz}$, $1 \mathrm{H}) ; 3.29-3.36(\mathrm{~m}, 3 \mathrm{H}) ; 3.39\left(\mathrm{dd}, J_{1}=14.7 \mathrm{~Hz}, J_{2}=3.7 \mathrm{~Hz}, 1 \mathrm{H}\right)$; $3.47-3.53(\mathrm{~m}, 2 \mathrm{H}) ; 3.56\left(\mathrm{dd}, J_{1}=15.2 \mathrm{~Hz}, J_{2}=6.4 \mathrm{~Hz}, 1 \mathrm{H}\right) ; 3.92$ $(\mathrm{q}, J=7.0 \mathrm{~Hz}, 2 \mathrm{H}) ; 5.21(\mathrm{~d}, J=10.1 \mathrm{~Hz}, 1 \mathrm{H}) ; 5.26(\mathrm{~d}, J=17.0 \mathrm{~Hz}$, $1 \mathrm{H}) ; 5.64$ (bt, $J=5.4 \mathrm{~Hz}, 1 \mathrm{H}) ; 5.88-5.98(\mathrm{~m}, 1 \mathrm{H}) ; 7.02$ (d, $J=7.3$ $\mathrm{Hz}, 1 \mathrm{H}) ; 7.23$ (bs, $1 \mathrm{H}) ; 7.27$ (t, $J=7.8 \mathrm{~Hz}, 1 \mathrm{H}) ; 7.72(\mathrm{~d}, J=8.3 \mathrm{~Hz}$, $1 \mathrm{H}) ; 9.33$ (bs, $1 \mathrm{H}) .{ }^{13} \mathrm{C} \mathrm{NMR}\left(\mathrm{CDCl}_{3}, 125 \mathrm{MHz}\right) \delta 15.3,15.9,26.5$, 27.2, 29.4, 31.3, 35.9, 39.6, 40.1, 42.3, 45.3, 55.6, 56.4, 57.3, 63.4, 112.0, 115.9, 116.5, 118.2, 119.1 125.4, 128.2, 132.8, 133.1, 133.4, 152.4 155.0, 177.9; HRMS (ESI): $m / z$ calcd for $\mathrm{C}_{29} \mathrm{H}_{43} \mathrm{~N}_{6} \mathrm{O}_{3}:[\mathrm{M}+$ $\mathrm{H}]^{+}, 523.3391$; found: 523.3385 .

\section{Acknowledgements}

Financial support for this research was provided by Lek d.d., a Sandoz Company. We would like to acknowledge Roman Burja TLCM head for valuable help, Dr. Martin Črnugelj for NMR assistance and University of Ljubljana, Faculty of Chemistry and Chemical Technology for high resolution mass analyses and elemental microanalyses. Dr. Franc Požgan gratefully acknowledges The Ministry of Higher Education, Science and Technology of the Republic of Slovenia, the Slovenian Research Agency (P1-0230-0103) and EN $\rightarrow$ FIST Centre of Excellence for their partial financial support.

\section{Notes and references}

1 P. Del Dotto and U. Bonuccelli, Clin. Pharmacokinet., 2003, $42,633$.

2 ICH Q3A Guideline, Revision 2, 2008.

3 European Pharmacopoeia, Monograph C, 7th edn (7.5), 2012, p. 1535.

4 (a) E. Brambilla, E. di Salle, G. Briatico, S. Mantegani and A. Temperilli, Eur. J. Med. Chem., 1989, 24, 421; (b) P. Salvati, A. M. Caravaggi, A. Temperilli, G. Bosisio, O. Sapini and E. di Salle, US Pat. 4526892, 1985, Chem. Abstr., 1985, 103, 160754.

5 (a) A. Stoll and A. Hofmann, Helv. Chim. Acta, 1943, 26, 2070; (b) A. Stoll, A. Hofmann and Th. Petrzilka, Helv. Chim. Acta, 1946, 29, 635; (c) R. Ručman and N. Djordjevič, DE Pat. 2708042, 1976, Chem. Abstr., 1977, 88, 158466. 
6 G. Marzoni, W. L. Garbrecht, P. Fludzinski and M. L. Cohen, J. Med. Chem., 1987, 30, 1823.

7 J. V. Braun, K. Heider and E. Müller, Chem. Ber., 1918, 51, 273. 8 (a) A. M. Crider, R. Grubb, K. A. Bachmann and A. K. Rawat, J. Pharm. Sci., 1981, 70, 1319; (b) T. A. Montzka, J. D. Matiskella and R. A. Partyka, Tetrahedron Lett., 1974, 15, 1325; (c) Z. Časar and T. Mesar, WO Pat. 2009133097, 2009, Chem. Abstr., 2009, 151, 528967.

9 E. C. Kornfeld and N. J. Bach, EP Pat. 3667, 1979, Chem. Abstr., 1979, 92, 181450.

10 M. Hafner, M. Sulyok, R. Schuhmacher, C. Crews and R. Krska, World Mycotoxin J., 2008, 1, 67.

11 J. Beneš, A. Černý, V. Miller and S. Kudrnáč, Collect. Czech. Chem. Commun., 1983, 48, 1333.

12 Amount of epimerisation in transformation of 8 to 9 was determined on the worked up, crude reaction mixture. Recording ${ }^{1} \mathrm{H}$ NMR of the reaction mixture showed on $6: 1$ epimer ratio. Triplets of $\mathrm{CH}_{3} \mathrm{CH}_{2} \mathrm{NCO}$ at $\delta$ [ppm] 1.15 and 1.12 of $9 a$ and $9 b$ were used. See ESI. $\dagger$

13 S. Ohno, Y. Adachi, M. Koumori, K. Mizukoshi, M. Nagasaka and K. Ichihara, Chem. Pharm. Bull., 1994, 42, 1463.

14 R. Gažák, V. Křen, P. Sedmera, D. Passarella, M. Novotna and B. Danieli, Tetrahedron, 2007, 63, 10466.

15 Z.-X. Wang, Y. Q. Li, M. Kondamreddy and X. Cai, US Pat. 20080275240, 2008, Chem. Abstr., 2008, 149, 534450.

16 (a) S. Mantegani, E. Brambilla, C. Caccia, E. di Salle, M. A. Cervini, R. A. McArthur, G. Traquandi and M. Varasi, Il Farmaco, 1998, 53, 65; (b) L. Bernardi, S. Mantegani, A. Temperili and G. Traquandi, GB Pat. 2162173, 1986, Chem. Abstr., 1986, 105, 79216.

17 K. E. Pfitzner and J. G. Moffatt,J. Am. Chem. Soc., 1963, 85, 3027. In general DCC was used for DMSO activation, albeit more expensive $\mathrm{EDC} \cdot \mathrm{HCl}$ was also employed. There were no differences in reaction yield between the two, although aldehyde prepared using $\mathrm{EDC} \cdot \mathrm{HCl}$ did not require so thorough purification with respect to the residual urea byproduct.

18 J. R. Parikh and W. v. E. Doering, J. Am. Chem. Soc., 1967, 89, 5505.
19 (a) P. A. Stadler and R. K. A. Giger, in Natural Products and Drug Development, ed. P. L. Krogsgaard, S. C. Brøgger and H. Kofod, Munksgard, Copenhagen, 1984, pp. 463484; (b) P. M. Dewick, in Medicinal Natural Products, John Wiley and Sons, Ltd, 2nd edn, 2002, pp. 368376.

20 S. Inuki, A. Iwata, S. Oishi, N. Fujii and H. Ohno, J. Org. Chem., 2011, 76, 2072.

21 (a) B. O. Lindgren and T. Nilsson, Acta Chem. Scand., 1973, 27, 888; $(b)$ B. S. Bal, W. E. Childers, Jr and H. W. Pinnick, Tetrahedron, 1981, 37, 2091.

22 (a) B. F. DeTar and R. Silverstein, J. Am. Chem. Soc., 1966, 88, 1013; (b) J. Podlech, Synthesis of Peptides and Peptidomimetics, in Houben-Weyl, Methods of Organic Chemistry, Additional and Supplementary Volume E22a to the $4^{\text {th }}$ Edition, ed. M. Goodman, A. Felix, L. Moroder and C. Toniolo, Georg Thieme Verlag, Stutgart, New York, 2001, pp. 517-533.

23 (a) S. W. Ashford, K. E. Henegar, A. M. Anderson and P. G. M. Wuts, J. Org. Chem., 2002, 67, 7147; (b) A. L. Gutman, G. Nisnevich, I. Rukhman, B. Tishin, A. Vilensky and B. Pertsikov, US Pat. 20020177709, 2002, Chem. Abstr., 2002, 138, 4728; (c) J. Galambos, L. Czibula, F. Sebõk, S. Bálint and F. Kassai, WO Pat. 2005085243, 2005, Chem. Abstr., 2005, 143, 286587.

24 A solvent screening was made by others (see ref. 15) with the aim to improve the ratio of cabergoline (1) vs. its regioisomer in the reaction of cabergolinic acid A with $\mathrm{EDC} \cdot \mathrm{HCl}$. The best results were achieved with halogenated solvents like DCM, DCE and chlorobenzene with the ratios of cabergoline (1) vs. regioisomer of $4.1,4.1$ and 4.7 respectively.

25 Ratio of $\mathbf{C} v s .15$ was determined from ${ }^{1} \mathrm{H}$ NMR spectrum of evaporated crude reaction mixture. Singlets of $\mathrm{NMe}_{2}$ at $\delta$ [ppm] 2.30 and 2.28 for $\mathbf{C}$ and $\mathbf{1 5}$ were used.

26 I. Candiani, W. Cabri, F. Zarini, A. Bedeschi and S. Penco, Synlett, 1995, 605.

27 T. Fehr, P. A. Stadler and A. Hofmann, Helv. Chim. Acta, 1970, 53, 2197. 